\title{
Disorder-Induced Transformation of the Energy Landscapes and Magnetization Dynamics in Two-Dimensional Ensembles of Dipole-Coupled Magnetic Nanoparticles
}

\author{
David Gallina* and G. M. Pastor® \\ Institut für Theoretische Physik, Universität Kassel, Heinrich-Plett-Straße 40, 34132 Kassel, Germany
}

(Received 27 December 2019; revised manuscript received 16 March 2020; accepted 20 April 2020; published 25 June 2020)

\begin{abstract}
The interaction-energy landscapes (ELs) and magnetization dynamics of two-dimensional ensembles of dipole-coupled magnetic nanoparticles are theoretically investigated. Extended nanostructures are modeled by considering nonoverlapping nanoparticles (NPs) in a square unit cell with periodic boundary conditions. The local minima and connecting transition states of the EL are determined systematically for representative NP arrangements having different degrees of disorder. The topology of the ergodic networks of stationary points is analyzed from both local and energy perspectives by using kinetic networks and disconnectivity graphs. We show that increasing the degree of disorder not only increases, most significantly, the number of local minima and transition states but also changes the shape of the EL in a very profound way. While slightly disordered ensembles correspond to good structure seekers, which are funneled towards the global minima, strongly disordered systems show very rough landscapes with multiple low-energy local minima separated by relatively large energy barriers. The consequences of this transition on the long-time Markovian dynamics of the nanostructures are quantified by calculating the field-free magnetic relaxation after saturation and after quenching. The simulations indicate that the relaxation of weakly disordered systems follows a slightly stretched exponential law, with a single characteristic timescale for a wide range of temperatures. In contrast, strongly disordered systems show a much more complicated relaxation dynamics involving multiple timescales, slowing down and trapping, which is reminiscent of spin glasses.
\end{abstract}

DOI: 10.1103/PhysRevX.10.021068

Subject Areas: Magnetism, Nanophysics,

Statistical Physics

\section{INTRODUCTION}

Two-dimensional (2D) ensembles of magnetic nanoparticles (NPs) have been the focus of remarkable research activity in the past years [1-18]. From a fundamental perspective, these systems constitute an extremely challenging research topic, in which reduced dimensionality, competing interactions, and disorder merge, leading to novel collective behaviors. From a technological standpoint, magnetic nanostructures are most relevant for a variety of applications, particularly in the field of spintronics, memory devices, and high-density data storage $[19,20]$. A large number of experimental methods have been developed in order to fabricate NP ensembles, which can be divided into two main categories. In top-down methods, the nanostructures are created starting from uniform bulk or film samples, for instance, by means of lithography or edging [7].

\footnotetext{
*dg@physik.uni-kassel.de
}

Published by the American Physical Society under the terms of the Creative Commons Attribution 4.0 International license. Further distribution of this work must maintain attribution to the author(s) and the published article's title, journal citation, and DOI.
In contrast, in bottom-up approaches, the NPs are first synthesized by controlling the nucleation mechanisms, for example, in vacuum by using a cluster-beam source $[1,2,8,9]$, on clean surfaces by diffusion-controlled aggregation $[3,21]$, or in solution by organometallic chemical reactions [5]. These particles, which may be protected by a ligand shell or coated with a nonmagnetic material, are then deposited on a substrate or integrated in a three-dimensional matrix. From a microscopic perspective, it is important to remark that the different fabrication processes yield very different structural arrangements of the NPs in the nanostructures. These can range from a well-defined long-range order, as in some lithographic samples and auto-organized materials, to highly disordered samples with nearly random positions of the NPs, as in surface nanostructures obtained from cluster-beam deposition. It is one of the goals of this paper to demonstrate how the degree of disorder in the NP arrangement conditions the collective dynamical response of the ensemble in a most significant way.

The magnetic properties of nanostructured materials are known to depend on the size and composition of the NPs, on the surface coverage, and also on the geometrical arrangement of the particles. On the one hand, the originality resides in the specific behavior of individual clusters, 
including the effects of reduced size, low dimensionality, and type of environment (e.g., ultrahigh vacuum, metallic support, ligand shell, etc.). On the other hand, one would like to understand and control the organization and global properties of NP ensembles regarded as a whole. From the latter perspective, diluted and densely packed samples are fundamentally different. In weakly interacting ensembles (low coverage), the magnetic properties of the nanostructures are dominated by local and short-range effects, such as cluster-substrate hybridizations and magnetic anisotropy energies. The long-time dynamics is governed by singleparticle local-moment fluctuations, which are described by superparamagnetic Arrhenius-type models [22-24]. In this case, it is generally expected that a perturbative or mean-field treatment of the interparticle interactions could be an appropriate starting point and that the structural organization of the particles in the sample should not be so important qualitatively. In contrast, for strongly interacting ensembles (high coverage), the single-particle viewpoint is no longer meaningful. In this case, the change in the magnetization direction of any given particle inevitably induces changes in the orientation of the magnetic moments of the neighboring particles. Therefore, a cooperative response is expected whenever the interparticle couplings are comparable to or stronger than the local anisotropies, even though the details of the physical behavior should depend on the nature of the interactions (e.g., dipolar, Ruderman-Kittel-Kasuya-Yosida, direct exchange, etc.) and on the underlying NP arrangements (e.g., square, triangular, kagome, etc.). As a result, even the most elementary transitions-for example, the fluctuations between two adjacent metastable states or local minima (LM) in the energy landscape-involve collective changes of the magnetization directions of many NPs at the same time [25-27]. Consequently, the many-body behavior of the nanostructure needs to be taken into account from the start.

Previous experimental and numerical studies of twodimensional ensembles of interacting magnetic nanoparticles have revealed a variety of remarkable physical phenomena, including long-range-order phase transitions, continuous ground-state degeneracies, and order-bydisorder effects $[17,18,28-32]$. In this context, it is particularly interesting to investigate the magnetic response of NP ensembles to external perturbations, which may reveal unusual nonequilibrium phenomena, such as dynamical slowing down, ergodicity breaking, memory effects, and aging. Indeed, there is experimental and theoretical evidence that dense magnetic NP ensembles may show such remarkable properties [33-38], in which case one denotes them as superspin glasses because of the analogy to atomicspin glasses $[39,40]$. At the origin of these effects, one usually finds competing frustrating interactions and disorder, which lead to rough energy landscapes showing intricate networks of local minima separated by large, broadly distributed energy barriers [39-41]. Under these circumstances, the structural arrangement of the NPs plays a central role since it conditions the shape of the underlying interaction-energy landscape and all the resulting observable properties, including magnetic order, thermodynamics, and nonequilibrium dynamics.

The lack of symmetry resulting from disorder and the anisotropic long-ranged nature of dipolar interactions pose a serious challenge to theoretical investigations of magnetic nanostructures. Analytical approaches are, in general, very difficult or simply unfeasible. Hence, microscopic insight is usually obtained by means of numerical simulations [42]. So far, there have been a number of theoretical studies of the macroscopic response of NP ensembles, such as fieldcooled and zero-field-cooled magnetization curves, linear and nonlinear susceptibilities, and magnetic relaxation rates [43-46]. Moreover, elementary transitions between local minima have been studied by solving the Langevin dynamics and by using path-integral methods [47-49]. Nevertheless, many aspects of the most challenging collective behavior of magnetic nanostructures remain unexplored, particularly concerning the role of disorder [6,32,50-55]. Previous investigations have shown that structural disorder significantly changes the energy landscape of dipole-coupled magnetic NP ensembles by increasing the number of its local minima and reducing the correlation length or spatial extension of the elementary relaxation processes [25-27]. Although these results are exciting, they are also far from conclusive. Detailed investigations on how the local minima of the EL are connected with each other in order to form a network of metastable states are lacking. Little or no information is available on the topology of these important kinetic networks and on the timescales governing the dynamics within them. Furthermore, from an energy viewpoint, it remains unclear how the local basins of attraction and the accessible regions in configurational space merge as the available energy increases and barriers can be progressively overcome. This lack of knowledge is hampering since having a detailed picture of the organization of the energy landscapes of these complex systems is crucial in order to understand their equilibrium and dynamic properties from a microscopic perspective.

The main goals of this paper are to characterize the energy landscapes of dipole-coupled ensembles of magnetic NPs as a function of the orientation of the local magnetic moments and to quantify the corresponding magnetic relaxation dynamics. We pay particular attention to establishing the correlations between the degree of disorder, the properties of the landscape, and the observed dynamics. In Sec. II, the theoretical background is presented. Aside from the microscopic model describing the NP ensembles, we outline the methods used for identifying the distinctive features of the ELs and for calculating the corresponding Markovian relaxation dynamics. In Sec. III, the properties of representative ensembles having different 
degrees of disorder are presented and discussed in some detail. The topography of the ELs and, in particular, the organization of the networks of their stationary points [(local minima and transition states (TS)] are analyzed from both local and energy perspectives. The properties of magnetic NP ensembles are contrasted with those of other relevant complex systems, including ferromagnetic (FM) periodic models, dipolar-bonded clusters, and structural glasses. The field-free time evolutions of the magnetic order of the ensembles are determined starting from saturation or thermal quenching. The correlations between the observed dynamics and the underlying ELs are established. A remarkable transition from rapid, good-structureseeker relaxation to trapped glasslike behavior is revealed as the degree of disorder increases. Finally, in Sec. IV, we summarize our conclusions and point out some of the possible extensions and implications of this study.

\section{THEORY}

\section{A. Nanostructure model}

In order to describe the 2D ensembles of interacting NPs, we consider nonoverlapping particles that are contained in a square unit cell with periodic boundary conditions. Because of the strong interatomic exchange couplings within the NPs, and taking into account their small size with diameters of the order of 5-10 nm, it is safe to regard each particle $k$ as a monodomain and to characterize its magnetic state by a classical moment $\vec{\mu}_{k}$ with a fixed module $[8,9,56]$. The present model extends the one proposed in Refs. [25-27] by allowing for a fully unconstrained three-dimensional (3D) orientation of all $\vec{\mu}_{k}$. In this way, any possible artifacts resulting from the previously imposed in-plane constraint of $\vec{\mu}_{k}$ (e.g., overestimation of the energy barriers, distorted minimum energy paths, etc.) are removed. Having access to the complete magnetic configurational space of the ensemble is crucial in order to determine the entropy associated with the statistical fluctuations at the local minima and transition states. They are important for calculating the transition rates between the metastable states that define the dynamics of the system (see Sec. II E). Different geometrical arrangements of the NPs are considered in order to investigate the role of autoorganization and disorder on the magnetic behavior of the ensembles: (i) weakly to moderately disordered squarelattice structures, (ii) weakly to moderately disordered triangular-lattice structures, and (iii) completely random distributions of nonoverlapping particles. In the case of the square and triangular structures, disorder is introduced by displacing the particles from their regular periodic positions according to a Gaussian distribution with standard deviation $\sigma_{r}$. More than 200 different disordered nanostructures have been investigated in detail. Representative examples of these structures are given in Sec. III A.

In this paper, we focus on dipole-coupled ensembles of magnetic NPs, which correspond to the limit of nearly spherical particles having weak local anisotropy [23]. These strongly interacting systems are particularly challenging since they show noncollinear metastable magnetic configurations and collective transitions, in which the orientations of the moments of many different NPs change at the same time $[27,50-53,55]$. Given the locations $\vec{r}_{k}$ and magnetic moments $\vec{\mu}_{k}$, the dipolar energy of the system is given by

$$
E=\frac{\mu_{0}}{8 \pi} \sum_{k \neq l}\left[\frac{\vec{\mu}_{k} \cdot \vec{\mu}_{l}}{r_{k l}^{3}}-\frac{3\left(\vec{\mu}_{k} \cdot \vec{r}_{k l}\right)\left(\vec{\mu}_{l} \cdot \vec{r}_{k l}\right)}{r_{k l}^{5}}\right],
$$

where $\vec{r}_{k l}=\vec{r}_{k}-\vec{r}_{l}$ is the vector connecting the centers of particles $k$ and $l, r_{k l}=\left|\vec{r}_{k l}\right|$ the corresponding distance, and $\mu_{0}$ the vacuum permeability. Since the particles are usually protected by ligands and do not overlap, there are no metalmetal contacts and thus no contributions from directexchange interactions between the NP moments. The orientation of $\vec{\mu}_{k}$ is defined by the polar and azimuthal angles $\theta_{k}$ and $\phi_{k}$. Hence, each magnetic configuration is characterized by a set of $N$ pairs of angles $\left\{\theta_{k}, \phi_{k}\right\}$ in the unit cell $(k=1, \ldots, N)$. Extending the model by including other types of magnetic interactions (quadrupolar, direct or indirect exchange, etc.) or local anisotropy fields is straightforward.

\section{B. Energy landscapes}

The static and dynamic properties of classical manybody systems are conditioned by the underlying energy landscape (EL) as a function of the relevant degrees of freedom-in the present case, the magnetic moments $\vec{\mu}_{k}$. The EL can be characterized by its remarkable configurations or stationary points, namely, the LM and TS [41]. Although the focus on the stationary points and the associated basins of attraction is a meaningful simplification, locating and cataloging all of them remains a serious challenge for complex systems having high-dimensional configurational spaces. Therefore, one usually aims to construct a representative sample of LM and TS relevant for the dynamics of the system under study $[41,57,58]$.

The network of stationary points is determined in the following way [59]: (i) One considers the magnetic configuration of a local minimum, for example, the global minimum, and from there, one starts an eigenvectorfollowing search for a nearby TS along one of the eigenvectors with the lowest eigenvalue of the Hessian matrix $\mathbf{H}$ at this minimum [58]. (ii) Once a TS is found, the two LM connected by it are identified by performing two limited-memory Broyden-Fletcher-Goldfarb-Shanno (L-BFGS) minimizations [60] starting at the configurations located at both sides of the recently found first-order saddle point. Each triplet obtained in this way-the two LM and the connecting TS-defines an elementary transition or relaxation process. In almost all cases, the triplet is connected to the already-recorded network of stationary 
points since at least one local minimum is known, namely, the starting one. (iii) The triplet LM-TS-LM is then added to the database of stationary points, unless it was already known. Triplets that are disjoint from the existing network are disregarded at this stage. (iv) The algorithm proceeds by starting the eigenvector-following TS search along a different eigenvector of $\mathbf{H}$. Once a predefined number of eigenvectors of $\mathbf{H}$ at the given minimum have been tried (typically, 15-20 searches are enough to find most of the adjacent TS), one moves to the next local minimum in the database, which amounts to returning to step (i). The algorithm terminates after all local minima in the database have been explored or after a specified number of connected LM have been found.

\section{Kinetic networks}

The calculated LM and TS form a connected or ergodic network, usually known as the kinetic network of the EL. It can be naturally represented by an undirected graph, in which the nodes symbolize the LM and the edges signify the connecting pathways going through the TS $[61,62]$. Examples of these networks are given in Sec. III B. The topology of the network of stationary states can be characterized by a number of local and global properties. A fundamental local property of each node $i$ is the degree or number of connections $N_{c}(i)$, which represents the number of links between this node and any other node of the network. Since different networks often vary widely in size, it is meaningful to introduce the local connectivity density

$$
\rho_{c}(i)=\frac{N_{c}(i)}{N_{\mathrm{LM}}-1},
$$

given by the ratio between $N_{c}(i)$ and its maximum possible value, where $N_{\mathrm{LM}}$ is the total number of LM or nodes in the network. Thus, $\rho_{c}(i)$ measures the relative importance of the LM $i$ in the kinetic network [61,62]. The LM with large $\rho_{c}$ are referred to as hubs since they are connected to a significant fraction of all other LM. In the present work, we consider that a LM $i$ is a hub when $\rho_{c}(i) \geq 0.1$. Hubs play a central role in the dynamics of complex systems because they participate in most relaxation processes. If the transition rates are favorable, they may funnel and speed up the dynamics, while in the opposite case, they are responsible for trapping and slowing down. Their number, connectivity, and the height of the barriers surrounding them are therefore crucial for determining the dynamics and its timescales.

Another fundamental property of the network of stationary points is the distance $d_{i j}$ between the LMs $i$ and $j$, which is equal to the smallest number of elementary LMTS-LM transitions or segments needed to connect them. The average path distance $\bar{d}$ over all pairs of LMs quantifies the extension of the network in units of an elementary relaxation step $[61,62]$. When $\bar{d}$ is small, the system has to undergo fewer elementary transitions in order to relax from an arbitrary excited state to its equilibrium configuration. In contrast, as $\bar{d}$ increases, the relaxation mechanisms become more complex and usually slower since a larger number of intermediate TS are involved. In this context, it is useful to introduce the eccentricity $\epsilon_{i}$ of node $i$ as the largest $d_{i j}$ between this node and any other node $j$. Thus, $\epsilon_{i}$ quantifies the extent of the network from the perspective of node $i$. From the set of all $\epsilon_{i}$, one derives the radius $R$ and diameter $D$ of the network as the smallest and largest $\epsilon_{i}$, respectively $[61,62]$. Here, $R$ and $D$ are global network characteristics, whose values increase, together with the average distance $\bar{d}$, as the complexity of the network increases and the approach to equilibrium becomes more intricate. However, notice that $R$ and $D$ are given by the local environment of the two particular nodes giving the extrema. They are therefore more prone to fluctuations and may be less representative than $\bar{d}$.

Besides the connectivity density $\rho_{c}(i)$, it is also interesting to investigate how the neighbors of a node are connected with each other in order to identify the presence of subgroups or clusters of nodes among which direct transitions are possible. This information, known as clustering of the network, can be used to anticipate rapid dynamics within subgroups of basins or metabasins. A global measure of the degree to which the neighbors of a node are also neighbors of each other is given by the transitivity

$$
C=3 \times \frac{\text { number of triangles }}{\text { number of triads }}
$$

which represents the probability that in a triad of nodes, in which $i$ is connected to $j$ and $j$ to $k, i$ and $k$ are also connected [63]. The factor 3 in Eq. (3) takes into account that each triangle contains 3 triads.

The above network metrics are very useful in order to assess the properties of magnetic nanostructures, for example, as a function of the NP arrangement and degree of disorder. They also allow quantitative comparisons with the behavior of other complex systems in very different physical situations (glasses, atomic clusters, proteins, etc.). Two idealized model networks appear as important references in this context since they are representative of extreme-opposite characteristics. On the one hand, we have the random networks, in which the nodes are connected randomly with a given average degree [64]. These networks show relatively short average distances $\bar{d}$ and very low transitivities $C$. On the other hand, we have lattice networks, which correspond to the nearest-neighbor (NN) map of compact lattice structures and which have comparatively large values of both $\bar{d}$ and $C$ [61,62]. Finally, a recurring question in network analysis is to determine whether the considered system shows small-world properties [65]. In a small-world network, the probability that the 
neighbors of a node are also neighbors of each other is high, and the number of elementary transitions or edges needed to connect any two nodes is small. Therefore, following Watts and Strogatz [65], we consider that a network shows small-world behavior if it combines a small value of $\bar{d}$, which is comparable to or smaller than the one of a random network having the same size and average degree, with a significant value of $C$, which is typical of compact-lattice networks. These properties are often associated with the presence of hubs and can be found in many practical examples such as neural, airport, and electric-power-grid networks $[61,62,65]$.

\section{Disconnectivity graphs}

While the topology of the kinetic networks and the parameters characterizing them provide a clear picture of how the basins or metastable states are connected among each other from a local or configurational perspective, they give no insight on the distribution of the stationary points from an energetic point of view. A complementary approach that removes this limitation is to determine the corresponding disconnectivity graphs (DGs) [66], examples of which are shown in Sec. III C. The procedure to calculate the DGs is the following [41]: For any given energy $E$, one groups the LM of the ergodic network into disjoint sets called superbasins. Two LM are said to belong to the same superbasin if they can be connected by a path that never exceeds the energy $E$. In other words, reaching a minimum outside a given superbasin always requires energies that are larger than $E$. It is clear that the superbasins define equivalent classes. In the absence of degeneracies, there is only one superbasin at very low energies that contains the global minimum. However, if the ground state is $N$-fold degenerate, one finds $N$ disjoint superbasins at low $E$. As the available energy $E$ is gradually increased, more and more LM become accessible. Notice that these new minima are not necessarily connected with the global minimum or with each other for energies below $E$. Thus, new superbasins are, in general, identified (see, for example, Fig. 5 in Sec. III C). However, if $E$ is further increased, one also observes that, at some point, there are superbasins that start to merge since the energy barriers between them can be overcome. Finally, for very high $E$, only one superbasin is left. It contains all the LM of the system.

In practice, the disconnectivity graph is constructed by performing the above-mentioned analysis at a discrete set of typically equidistant energies, which are indicated on the $y$ axis of the graph (see, for example, Fig. 5) [41]. At each of these energies, a superbasin is represented by a node, taking into account that two nodes are connected if the corresponding superbasins share at least one common local minimum. The horizontal positions of the nodes in the graph are arbitrary a priori. For the sake of clarity, they are usually chosen by grouping superbasins that have similar energies and energy barriers. The result is a treelike graph, where the end of each branch corresponds to a single local minimum and its corresponding local basin of attraction (see Fig. 5) [41]. All the LM that are connected to a common node are mutually accessible at the corresponding energy and thus belong to the same superbasin. A particularly transparent classification of archetypal energy landscapesby exploiting the analogies between DGs and weepingwillow, palm, or banyan trees—may be found in Ref. [67].

\section{E. Markovian dynamics}

At low temperatures, the transitions between different metastable states or basins can be regarded as rare events, so the time evolution consists of a series of independent elementary transitions separated by relatively long thermalization periods. Under these conditions, memory effects are lost, and the dynamics can be regarded as Markovian. The transition rates $k_{j i}$ between the metastable states define the dynamics, which is governed by the master equation. In the framework of transition state theory (TST) [68], they are given by

$$
k_{j i}^{\mathrm{TST}}=\left\langle\delta[s(\mathbf{x})] v_{\perp}(\mathbf{x}) H\left[v_{\perp}(\mathbf{x})\right]\right\rangle_{T},
$$

where \langle\rangle$_{T}$ denotes the thermal average, $\mathbf{x}$ stands for the dynamical variables of the system (in the present case, the set of $N$ pairs of angles $\left.\left\{\theta_{k}, \varphi_{k}\right\}\right), s(\mathbf{x})=0$ defines the surface dividing the initial basin $i$ from the final basin $j, v_{\perp}(\mathbf{x})$ is the projection of the configurational velocity vector $\dot{\mathbf{x}}$ onto the normal of the dividing surface, and $H\left[v_{\perp}(\mathbf{x})\right]$ is a Heaviside function, taking the value 1 if $v_{\perp}(\mathbf{x})$ points towards the final state and zero otherwise [69]. This expression can be worked out by performing a quadratic expansion of the energy at the initial local minimum $\mathbf{x}_{\mathrm{LM}}$ and at the first-order saddle point $\mathbf{x}_{\mathrm{TS}}$ [70]. The system configuration and energy around the minimum can be expressed in terms of the Hessian eigenvectors $\mathbf{e}_{\kappa}$ and eigenvalues $\varepsilon_{\kappa}$ as $\quad \mathbf{x}=\mathbf{x}_{\mathrm{LM}}+$ $\sum_{\kappa} q_{\kappa} \mathbf{e}_{\kappa}^{\mathrm{LM}}$ and $E(\mathbf{q})=E_{\mathrm{LM}}+\sum_{\kappa} \varepsilon_{\kappa}^{\mathrm{LM}} q_{\kappa}^{2}$, where the normal coordinates $q_{k}$ describe the fluctuations. Analogous expressions hold at the saddle point. The dividing surface is then approximated by the hyperplane spanned by the $2 N-1$ stable modes $\mathbf{e}_{\kappa}^{\mathrm{TS}}(\kappa=2, \ldots, 2 N)$ having positive Hessian eigenvalues $\varepsilon_{\kappa}^{\mathrm{TS}}$ at the saddle point. The normal to the dividing surface, given by the single unstable mode $\mathbf{e}_{1}^{\mathrm{TS}}$ $\left(\varepsilon_{1}^{\mathrm{TS}}<0\right)$, can be regarded as a reaction coordinate from which $v_{\perp}=d q_{1} / d t$ follows [70].

In order to determine $v_{\perp}$, the dynamics of the coupled magnetic moments needs to be taken into account $[71,72]$. The underlying time evolution can be described by the Landau-Lifshitz-Gilbert equations [73]

$$
\frac{d \theta_{k}}{d t}=\frac{-\gamma}{M_{k} \sin \theta_{k}\left(1+\alpha^{2}\right)}\left(\frac{\partial E}{\partial \varphi_{k}}+\alpha \sin \theta_{k} \frac{\partial E}{\partial \theta_{k}}\right)
$$

and 


$$
\frac{d \varphi_{k}}{d t}=\frac{\gamma}{M_{k} \sin \theta_{k}\left(1+\alpha^{2}\right)}\left(\frac{\partial E}{\partial \theta_{k}}-\frac{\alpha}{\sin \theta_{k}} \frac{\partial E}{\partial \varphi_{k}}\right),
$$

where $\theta_{k}$ and $\varphi_{k}$ are the polar and azimuthal angles defining the direction of the magnetic moment $\vec{\mu}_{k}$ of NP $k, \gamma$ is the gyromagnetic ratio, and $\alpha$ is the damping parameter. Linearizing these equations for small $q_{\kappa}$, one obtains $v_{\perp}=$ $d q_{1} / d t=\sum_{\kappa} a_{\kappa} q_{\kappa}$ as a linear combination of the fluctuations $q_{k}$ within the dividing plane at the TS [71,72]. The resulting rate in harmonic transition state theory (HTST) is given by

$k_{j i}^{\mathrm{HTST}}=\frac{1}{2 \pi} \frac{J_{\mathrm{TS}}}{J_{\mathrm{LM}}} \frac{\prod_{\nu=1}^{2 N} \sqrt{\varepsilon_{\nu}^{\mathrm{LM}}}}{\prod_{\kappa=2}^{2 N} \sqrt{\varepsilon_{\kappa}^{\mathrm{TS}}}} \sqrt{\sum_{\lambda=2}^{2 N} \frac{a_{\lambda}^{2}}{\varepsilon_{\lambda}^{\mathrm{TS}}}} e^{-\left(E_{\mathrm{TS}}-E_{\mathrm{LM}}\right) / k_{B} T}$,

where $T$ refers to the temperature and $J_{\mathrm{LM}}=\prod_{\nu=1}^{N} \sin \theta_{\nu}^{\mathrm{LM}}$ $\left(J_{\mathrm{TS}}=\prod_{\kappa=1}^{N} \sin \theta_{\kappa}^{\mathrm{TS}}\right)$ to the Jacobian of the transformation to spherical coordinates [71,72]. With an appropriate choice of the local coordinates, the latter can be set to 1 . Notice that in the harmonic approximation, the transition rates take the form of an Arrhenius ansatz $k_{j i}=$ $f_{j i} e^{-\Delta E_{j i} / k_{B} T}$ for each pair of metastable states $i$ and $j$, where the prefactor $f_{j i}$ depends on $i$ and $j$ but not on $T$, and the Boltzmann factor corresponding to the activation energy $\Delta E_{j i}=E^{\mathrm{TS}}-E^{\mathrm{LM}}$ carries all the temperature dependence. Equation (7) takes into account the environment-specific entropy associated with the fluctuations of the magnetic configuration at the initial basin $i$ and at the TS leading to the basin $j$, as well as the probability flux $v_{\perp}$ at the dividing surface. The $k_{j i}$ obtained in this way are consistent with the energy landscape and the local curvatures at the stationary points. Thus, a rigorous quantification of the timescales of the relaxation processes is provided, including the distribution thereof throughout the landscape. Any ad hoc assumptions on $f_{j i}$ are thereby avoided. In practice, the values obtained in our calculations are in the range $f_{j i}=10^{8}-10^{11} \mathrm{~Hz}$ depending on the initial and final basins. These results are qualitatively in agreement with the figures inferred from experiment [74].

Once the transition rates $k_{i j}$ for all elementary transitions are obtained, the time evolution of the system is calculated by solving the master equation [75]

$$
\frac{d \mathbf{p}}{d t}=\mathbf{Q p}
$$

where the $i$ th component $p_{i}$ of the vector $\mathbf{p}$ represents the occupation probability of the minimum or basin $i$. The transition-rate matrix $\mathbf{Q}$ is given by

$$
\mathrm{Q}_{i j}=k_{i j}-\delta_{i j} \sum_{l=1}^{N_{\mathrm{LM}}} k_{l i}
$$

where $N_{\mathrm{LM}}$ is the total number of LM of the system. The solution of this linear equation takes the form

$$
\mathbf{p}(t)=e^{\mathbf{Q} t} \mathbf{p}(0),
$$

where $\mathbf{p}(0)$ stands for the initial configuration of the system. Expressing the matrix exponential in terms of the normalized eigenvectors $\mathbf{u}_{i}$ and eigenvalues $\lambda_{i}$ of $\mathbf{Q}$, one obtains

$$
\mathbf{p}(t)=\sum_{i=1}^{N_{\mathrm{LM}}} c_{i} e^{\lambda_{i} t} \mathbf{u}_{i}
$$

where $c_{i}=\mathbf{p}(0) \cdot \mathbf{u}_{i}$ is determined by the initial probability distribution [75]. Alternatively, Eq. (8) can be solved by using the kinetic Monte Carlo method [76,77].

\section{RESULTS AND DISCUSSION}

The first goal of this section is to investigate the interaction-energy landscapes of ensembles of magnetic NPs and, in particular, to quantify the effects of structural disorder. To this aim, a large number of model realizations of the nanostructures, more than 600 in all, have been explored. Three representative examples are illustrated in Fig. 1, which correspond to a weakly disordered square lattice, a weakly disordered triangular lattice, and a random distribution of nonoverlapping particles. Rather than performing statistical averages over a large number of NP arrangements, our aim here is primarily to analyze in detail the energy landscapes of representative nanostructures, in order to shed light on the role of disorder on the topology of the landscapes and on the dominant microscopic magnetic relaxation mechanisms. To quantify the effects of structural disorder, which is inherent to any experimental sample, we start from periodic square and triangular lattices and introduce random displacements of the NP positions according to a Gaussian distribution with mean-square deviation $\sigma_{r}$. The weakly disordered ensembles shown in Fig. 1 correspond to $\sigma_{r}=0.05 r_{0}$, where $r_{0}$ is the nearestneighbor distance of the periodic arrangement. Increasing $\sigma_{r}$ allows us to control the importance of disorder systematically. The limit of strongly disordered ensembles is reached when $\sigma_{r}$ is comparable to or larger than $r_{0}$, which corresponds to a random distribution of nonoverlapping nanoparticles.

For the calculations, we consider Fe particles having a diameter $\phi=3 \mathrm{~nm}$ and the structural arrangements shown in Fig. 1. In this size range, the Fe NPs are known to behave like ferromagnetic monodomains, provided that the temperature is not too high, since $\phi$ is orders of magnitude smaller than the width of any domain wall. Assuming, for simplicity, that the atomic magnetic moments and the density within the particles are bulklike $\left(\mu_{b}=2.2 \mu_{B}\right.$ and bcc lattice with $a=0.29 \mathrm{~nm}$ ), one finds that the total magnetic 


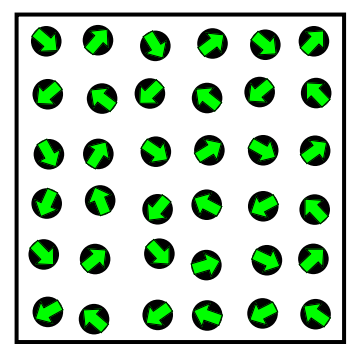

(a)

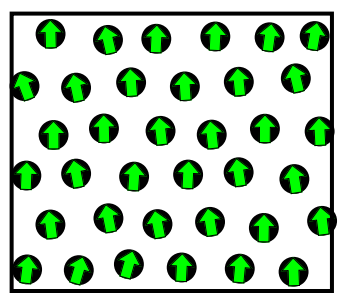

(b)

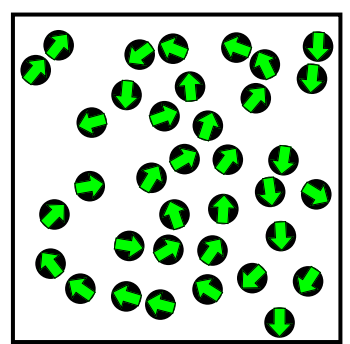

(c)

FIG. 1. Ground-state magnetic configurations of representative two-dimensional ensembles of nanoparticles: (a) weakly disordered square structure $\left(\sigma_{r} / r_{0}=0.05\right)$, (b) weakly disordered triangular structure $\left(\sigma_{r} / r_{0}=0.05\right)$, and (c) nonoverlapping randomly distributed particles. The unit cells are shown, on which periodic boundary conditions apply.

moment of the NPs is $|\vec{\mu}| \simeq 2.55 \times 10^{3} \mu_{B}$. The extended nanostructures are modeled with finite unit cells containing $N=36$ particles and imposing periodic boundary conditions. Moderate surface coverages $c=0.28$ for the square and random ensembles and $c=0.33$ for the triangular ensemble are assumed $\left(c_{\max }=\pi / 2 \sqrt{3}=0.91\right)$. As discussed below, reasonable changes in the sample parameters do not affect our conclusions. Results on the dependence of average properties on $N$ and $c$ may be found in Refs. [25-27].

\section{A. Ground-state magnetic order}

In Fig. 1, the ground-state magnetic configurations of three representative ensembles are shown. In all cases, the directions of the NP moments are almost perfectly within the $x y$ plane, as indicated by the arrows. The most stable configuration of weakly disordered square-lattice structures is similar to the so-called microvortex (MV) state, in which the direction of $\vec{\mu}_{k}$ alternates from site to site, forming an angle $\pm \alpha$ with the $x$ axis as we move along a column or row of the square lattice [see Fig. 1(a)] [28,78]. The presence of disorder becomes apparent through minor deviations from the perfect alternation. The optimal configuration is doubly degenerate as any other since the dipole interaction is invariant with respect to the inversion of all $\vec{\mu}_{k}$ [see Eq. (1)]. It is interesting to note that the MV state also yields the lowest energy when the lattice is perfectly periodic. However, in this case, the energy is independent of the tilting angle $\alpha$. The continuous degeneracy is a consequence of the $C_{4}$ rotational symmetry of the periodic square lattice, as can be demonstrated by group-theory arguments [79-81]. Remarkably, the weakest degree of disorder breaks this symmetry and lifts the continuous degeneracy completely, by singling out one or a few specific values of $\alpha$ and by keeping essentially the same type of long-range correlations between the $\vec{\mu}_{k}$ as in the periodic case. This behavior is usually referred to as an order-by-disorder effect $[28,82-84]$. Notice that time-inversion symmetry implies that the ground state is degenerate with the one obtained by reversing all spin directions. It is important to remark that the actual orientation of $\vec{\mu}_{k}$ in the ground state (i.e., the MV angle $\alpha$ according to which $\vec{\mu}_{k}$ alternates from site to site) depends strongly on the particular realization of the nanostructure, bearing no apparent correlation for different disordered arrangements.

The most stable magnetic configuration of the weakly disordered triangular ensembles is ferromagnetic, with only minor local deviations of the direction of $\vec{\mu}_{k}$ at different nanoparticles $k[85,86]$. An example is shown in Fig. 1(b). As in the square lattice, the long-range magnetic correlations of the periodic case, where $\vec{\mu}_{k}=\vec{\mu}$ for all $k$, are preserved. In the perfect triangular lattice, the energy $E$ is independent of the direction of $\vec{\mu}$ within the $x y$ plane since $E$ is a quadratic form of $\mu_{x}$ and $\mu_{y}$ and since the $(x, y)$ representation of the $C_{6}$ symmetry group is irreducible. Disorder removes the point-group symmetry and, with that, the continuous degeneracy by stabilizing a particular average main direction of the magnetic moments. The actual orientation of $\vec{\mu}$ depends, of course, on the specific locations of the NPs $[28,82-84]$.

Finally, turning our attention to strongly disordered ensembles, we find that all signs of long-range magnetic order have disappeared. Instead, the ground state shows short-range head-to-tail orientations of the moments, which reflect the tendency to magnetic flux closure. An example of a random NP arrangement is shown in Fig. 1(c). By sampling a large number of different locations of the NPs in the unit cell, we confirm that the orientation of the average magnetization per particle $\vec{m}$ is uniformly distributed within the $x y$ plane. The calculated average absolute value for $N=$ 36 particles in the unit cell of Fig. 1(c) is $\bar{m}=0.13 \mu$, and the standard deviation is $\sigma=0.11 \mu$, where $\mu$ stands for the NP magnetic moment. These values are somewhat smaller than the average $\sqrt{m^{2}}$ of $N$ randomly oriented moments $(m \simeq \mu / \sqrt{N}=0.17 \mu)$ which can be understood as the consequence of flux closure. Similar results are obtained for the average over all LM: $\bar{m}=0.1 \mu$ and $\sigma=0.1 \mu$. This randomness contrasts with the behavior of weakly disordered structures where long-range correlations, either of MV or FM type, are clearly preserved (see Fig. 1). Further 
details on the low-lying magnetic configurations in weakly and strongly disordered systems are discussed in Sec. III C.

\section{B. Kinetic networks}

The ensembles of dipole-coupled magnetic NPs have a large number of metastable states or basins, which are connected by diverse transition states. A pair of minima connected by a single intermediate saddle point constitutes an elementary transition or relaxation process. By extension, this term also applies to the minimum energy path (MEP), which links the minima along the gradientfollowing curve passing through the saddle point. From the point of view of the magnetic order, one observes that, for strictly planar dipole-coupled NP arrangements, the magnetic moments lie within the $x y$ plane all along the MEP, including the TS. Figure 2 shows the kinetic network, i.e., the network of LM and TS, for the weakly disordered square ensemble of Fig. 1(a). In this case, the number of local minima and transition states are $N_{\mathrm{LM}}=170$ and $N_{\text {TS }}=680$. For each LM, the elementary transitions that connect it to any other LM through a single first-order saddle point are indicated by a grey segment. This representation of the landscape is probably somewhat confusing since the number of direct connections is quite important. Not much physical understanding of the EL can be inferred in this way. The situation changes profoundly when one highlights (in black), for each minimum, the

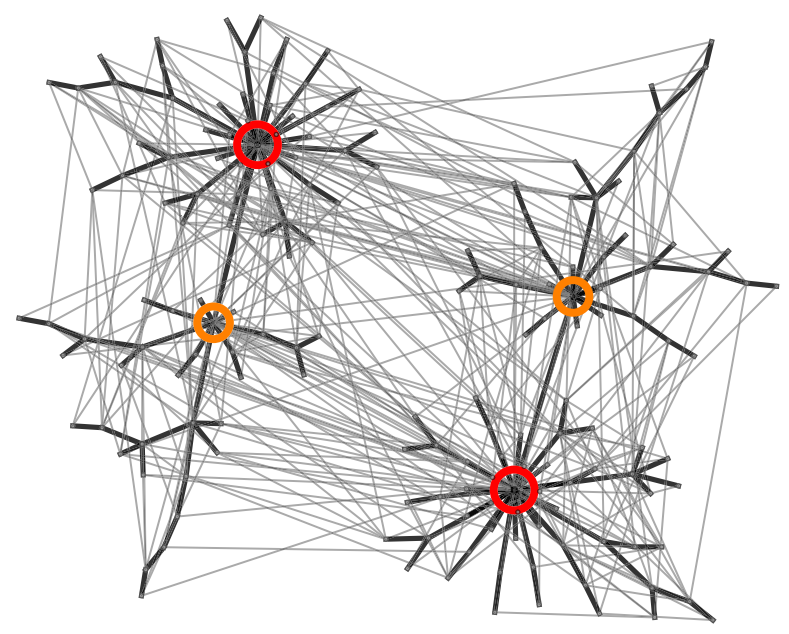

FIG. 2. Undirected kinetic network of the energy landscape of the weakly disordered square-lattice ensemble illustrated in Fig. 1(a). Each grey segment represents an elementary transition between two local minima through a first-order saddle point. The barrier heights and the lengths of the minimum energy paths connecting the minima are disregarded at this stage. The two degenerate ground states are indicated by red circles. Additional hubs $\left(\rho_{c}=0.33\right)$ of the kinetic network are indicated by orange circles. The thick black segments highlight the transitions that, starting from a given minimum, lead to a minimum having a lower or equal energy involving the smallest energy barrier [95]. transition that leads to a lower or equal energy and involves the lowest energy barrier. These are the dynamically dominant relaxation processes, at least in the lowtemperature limit where they yield the largest transition rates [70-72]. A much simpler and intelligible picture emerges. One observes that all the statistically relevant transitions are channeled towards the hubs, which act as veritable funnels of the magnetic configuration of the system. From this perspective, the ELs of weakly disordered ensembles share their main characteristics with other good-structure-seeking systems, such as magic number clusters, crystals, and good-folding proteins [87-94].

The network of LM in weakly disordered, square-lattice ensembles is centered around the twofold degenerate ground state and additional hubs. These states have huge basins of attraction and can be regarded as distortions of the continuously degenerate MV ground state of the periodic lattice. They are therefore a direct consequence of the symmetry breaking caused by disorder. For a given degree of disorder, the number of hubs depends, to some extent, on the specific location of the NPs. Values between 2 and 8 have been found for $\sigma_{r}=0.05 r_{0}$. Because of this hublike structure, the network of LM has a very small average path distance $\bar{d}=2.7$, diameter $D=6$, and radius $R=3$ (see Table I). The number of elementary transitions required to reach one of the ground states from any other state is thus very small. In addition, the transitions leading towards the ground states and hubs are mostly the ones with the smallest energy barrier. One concludes that in weakly disordered, square-lattice ensembles of magnetic NPs there

TABLE I. Topological parameters of the kinetic networks of stationary points. Results are given for the weakly disordered (WD) and strongly disordered (SD) nanostructures illustrated in Fig. 1, for the average of over more than 200 different arrangements of the magnetic NPs, and for a random network having the same number of nodes and edges: diameter $D$, radius $R$, average path distance between all nodes $\bar{d}$, and transitivity $C$. Distances are measured in numbers of elementary transitions. See also Figs. 2-4 and Sec. II C.

\begin{tabular}{lcccc}
\hline \hline Lattice & $D$ & $R$ & $\bar{d}$ & $C$ \\
\hline $\begin{array}{l}\text { WD square } \\
\text { ensemble }\end{array}$ & 6 & 3 & 2.7 & 0.11 \\
$\begin{array}{l}\text { Average } \\
\text { Random network }\end{array}$ & $5.4 \pm 0.6$ & $3.1 \pm 0.4$ & $2.7 \pm 0.2$ & $0.11 \pm 0.03$ \\
WD triangular & 4 & $4.0 \pm 0.0$ & $3.0 \pm 0.0$ & $0.04 \pm 0.01$ \\
$\quad$ ensemble & 2 & 2.3 & 0.06 \\
$\begin{array}{l}\text { Average } \\
\text { Random network }\end{array}$ & $5.5 \pm 0.5$ & $4.0 \pm 0.2$ & $3.3 \pm 0.0$ & $0.03 \pm 0.01$ \\
$\begin{array}{l}\text { SD ensemble } \\
\text { Average }\end{array}$ & $15.6 \pm 3.4$ & $10.0 \pm 1.9$ & $7.4 \pm 1.4$ & $0.10 \pm 0.02$ \\
Random network & $10.0 \pm 0.6$ & $6.9 \pm 0.3$ & $5.0 \pm 0.1$ & $0.00 \pm 0.00$ \\
\hline \hline
\end{tabular}




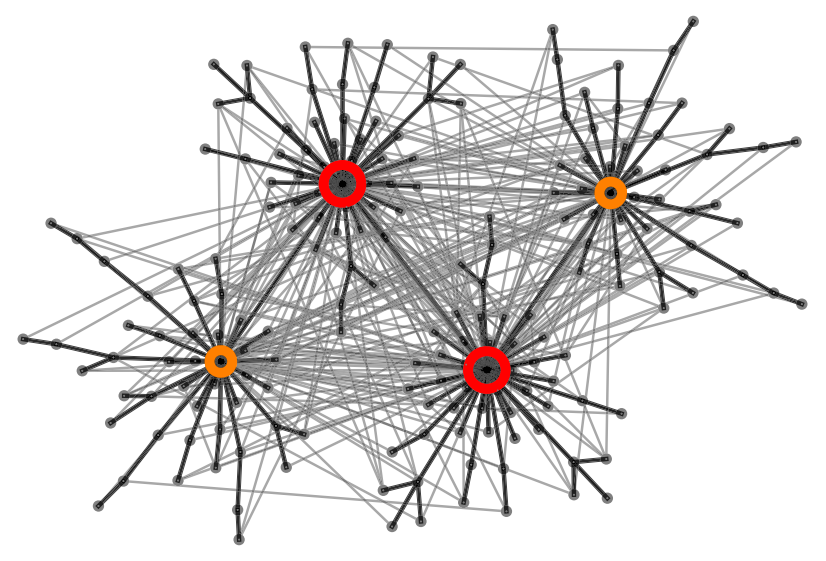

FIG. 3. Undirected kinetic network of the energy landscape of the weakly disordered, triangular NP ensemble illustrated in Fig. 1(b). The two degenerate ground states are indicated by the red circles. Additional hubs $\left(\rho_{c}=0.3\right)$ are indicated by orange circles. The thick black segments highlight the transitions that, starting from a given minimum, lead to a lower or equal energy involving the smallest energy barrier [95].

are no obstacles to a thermally activated relaxation dynamics, which should therefore be simple and relatively fast [96].

Figure 3 shows the kinetic network of the weakly disordered triangular ensemble illustrated in Fig. 1(b). The number of local minima $N_{\mathrm{LM}}=200$ and transition states $N_{\text {TS }}=822$ is larger than in the disordered square-lattice ensemble. However, the average path distance $\bar{d}=2.3$, diameter $D=4$, and radius $R=2$ are similar to or even somewhat smaller than in the square systems. These trends hold, in general, irrespectively of the precise geometrical realization or of the number of particles in the unit cell. Furthermore, for the square (triangular) lattice, the average over a large number of realizations with the same degree of disorder yields $\bar{N}_{\mathrm{LM}}=180$ and $\bar{N}_{\mathrm{TS}}=600\left(\bar{N}_{\mathrm{LM}}=230\right.$ and $\left.\bar{N}_{\text {TS }}=940\right)$ and average topological parameters $\langle\bar{d}\rangle=2.7$, $\langle R\rangle=3.1$, and $\langle D\rangle=5.4 \quad(\langle\bar{d}\rangle=2.5,\langle R\rangle=2.9$, and $\langle D\rangle=5.0)$. In the triangular case, the network of stationary points is also centered around the twofold degenerate ground state and additional hubs that stem from the continuously degenerate ground state of the periodic lattice. They preserve strong FM correlations, somewhat distorted as a result of the disorder-induced symmetry breaking. As already mentioned for the disordered square arrangements, the full network of LM and first-order SP is rather cumbersome and confusing. A clear picture emerges, however, when the most probable relaxation pathways are highlighted (black segments in Fig. 3). One observes, again, that the dominant transitions are systematically funneled towards the hubs. Weakly disordered triangular ensembles also have good structureseeking ELs and should therefore show unhindered relaxation properties.

A completely different physical picture emerges from the kinetic networks of strongly disordered ensembles. In Fig. 4, the kinetic network corresponding to the ensemble

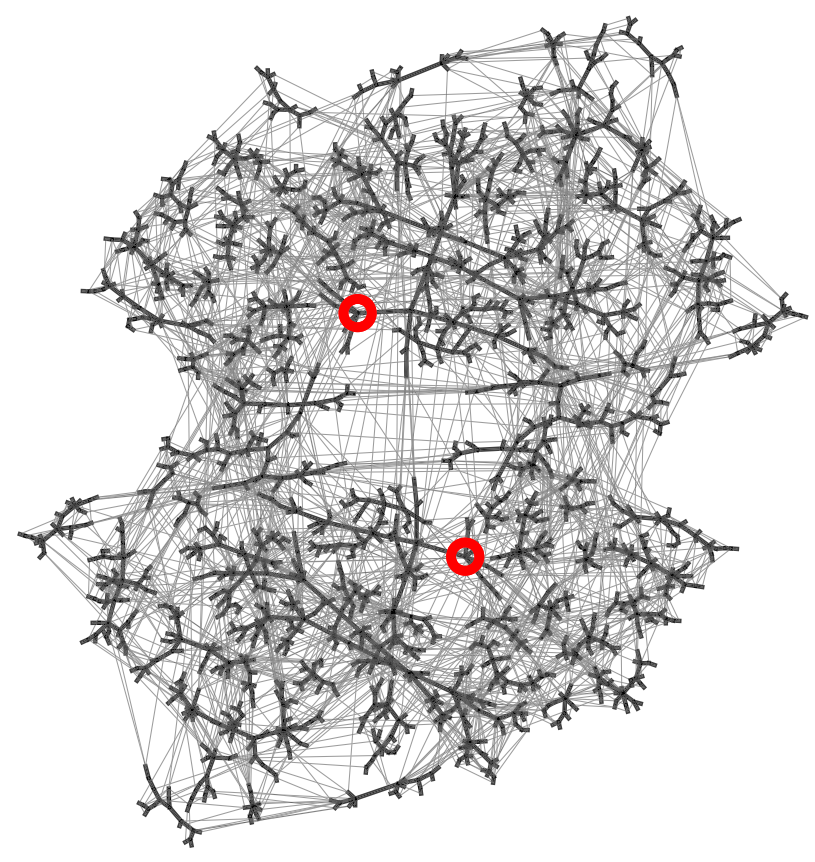

FIG. 4. Undirected kinetic network of the energy landscape of the strongly disordered ensemble illustrated in Fig. 1(c). The two degenerate ground states are indicated by the red circles. The thick black segments highlight the transitions that, starting from a given minimum, lead to a lower or equal energy involving the smallest energy barrier. Note that even the ground states do not qualify as hubs $\left(\rho_{c}=10^{-3}\right)$ [95].

illustrated in Fig. 1(c) is shown. In this case, one finds a remarkably large number of local minima $N_{\mathrm{LM}}=2810$ and transition states $N_{\mathrm{TS}}=8958$. Furthermore, the topography of the EL is far more complex, even if one focuses on the dominant transitions. The kinetic network is no longer centered around the two degenerate ground states. Instead, the network is quite dispersive, tending to decompose into a number of subgraphs. As a consequence, the random system has a much larger average path distance $\bar{d}=7.0$, diameter $D=14$, and radius $R=10$. Hence, the distances between the different local minima, as measured by the number of elementary transitions needed to connect them, are much longer, in general.

The remarkable increase in the number of LM in the strongly disordered system is consistent with the changes in the correlation length between the magnetic moments in the LM-TS-LM transitions [27]. In weakly disordered ensembles, the spatial extension of the change in magnetic configuration along the MEPs is quite large. This means that changes in the direction of a single magnetic moment induce changes in the direction of a large number of other magnetic moments, even if the latter are located at relatively large distances. Consequently, there are relatively few different metastable magnetic configurations since smaller parts of the ensemble cannot rearrange independently from each other. Because of the long correlation length, the metastable configurations need to match the 
symmetry of the underlying lattice. Thus, the average volume in configurational space of the attraction basins is comparatively large. In contrast, in strongly disordered systems, the correlation length between the magnetic moments is much shorter [27]. As a result, smaller domains of magnetic moments are able to rearrange fairly freely with respect to each other, which allows a wide variety of slightly different metastable configurations. Consequently, the volume per minimum and the average distance between them in configurational space are much smaller. A comparison of the basic properties of the networks of stationary points for different lattice structures and degrees of disorder is given in Table I.

It is interesting to take a closer look at the hubs of the various NP ensembles by analyzing how the local connectivity density $\rho_{c}$ of the most relevant and highly coordinated LM (i.e., the ground states and potential hubs) depends on the NP arrangement and degree of disorder [see Eq. (2)]. In the case of the weakly disordered ensembles, the values of $\rho_{c}$ for the ground states and hubs are substantial. For example, for the square lattice illustrated in Fig. 1(a), we have $\rho_{c}=0.35$ for the ground states and $\rho_{c}=0.22$ for the hubs $\left(N_{\mathrm{LM}}=170\right)$. Similarly, for the weakly disordered triangular lattice of Fig. 1(b), we find $\rho_{c}=0.47$ for the ground state and $\rho_{c}=0.3$ for the hubs $\left(N_{\mathrm{LM}}=230\right)$. Thus, the ground states and hubs are directly connected to an important fraction of all other LM of the network. These results are consistent with the picture inferred from the kinetic networks (Figs. 2 and 3) and with the fact that the basins of attraction of the hubs are extremely large. Random ensembles [e.g., Fig. 1(c)] behave profoundly different in this respect. The local connectivity density of the ground state is negligible: $\rho_{c}=0.001$, and no other LM qualifies as a hub $\left(N_{\mathrm{LM}}=2810\right)$. The most coordinated one has only $\rho_{c}=0.002$. According to our criterion for hub formation $\left(\rho_{c} \geq 0.1\right)$, there are no hubs in the strongly disordered systems, while there are four hubs in the considered weakly disordered systems.

The small-world behavior of the kinetic networks of good structure-seeking Morse clusters and structural-glass formers has been investigated in Ref. [97] by comparing their average path distance $\bar{d}$ and transitivity $C$ with those of random networks having the same average degree and number of LM. In this way, the authors were able to show that the networks of good structure-seeking clusters are small-world-like, whereas networks of the structural-glass formers are not [97]. Remarkably, magnetic NP ensembles show both contrasting behaviors, depending on the degree of disorder. Indeed, the kinetic networks of the weakly disordered, square (triangular) ensembles have a smaller average path distance $\bar{d}=2.7(\bar{d}=2.3)$ and a larger transitivity $C=0.11(C=0.06)$ than the corresponding random network, for which $\bar{d}=3.0$ and $C=0.04(\bar{d}=3.3$ and $C=0.03$ ). Thus, the weakly disordered arrangements show small-world properties. In contrast, for the strongly disordered NP ensemble of Fig. 1, we obtain $\bar{d}=7.0$, which is significantly larger than the corresponding random-network value $\bar{d}=5.1$. One therefore concludes that the strongly disordered NP ensembles do not have small-world kinetic networks. These results confirm that the former are good structure seekers while the latter show glassy behavior.

The changes in the topography of the landscape caused by structural disorder are crucial for the dynamical properties. In the weakly disordered ensembles, we have seen that the ground states and closely connected hubs can be regarded as the center of the energy landscape. Moreover, the dominant elementary transitions out of the excited states are all funneled towards them (see Fig. 2). These are the characteristics of a good structure-seeking behavior, in which very few intermediate relaxation steps are involved. In contrast, the relaxation mechanisms in strongly disordered ensembles are far more complex. If one looks at the dynamically relevant processes with the lowest energy barrier (highest rate), one sees that the graph breaks down into a set of poorly connected subgraphs. This result implies that the relaxation pathways become longer and, more important, that they are not systematically directed towards the ground states anymore. In contrast to the nearly periodic cases, the dynamics of strongly disordered ensembles can easily lead to very different magnetic configurations depending on the initial state. Consequently, the approach to equilibrium is likely to involve more than one characteristic timescale. At first, the system is expected to evolve rather rapidly within the subgraph or superbasin corresponding to its initial state, as conditioned by the dominant elementary transitions (i.e., the thick black segments in Fig. 4). Depending on temperature, this process can lead to a nearly thermalized distribution throughout the LM of the given subgraph. Subsequently, only on much slower stages does the relaxation between the different superbasins set in. The evolution towards thermal equilibrium, as implied by the ergodicity of our finite network of $\mathrm{LM}$, involves processes that become more and more rare. The relaxation is therefore expected to be hindered by trapping. This behavior, which is reminiscent of spin glasses, may lead to a breakdown of ergodicity in the thermodynamic limit [39]. The link between multiple relaxation timescales, broken ergodicity, glassy behavior, and multifunnel energy landscapes has been extensively studied for atomic and molecular clusters since they serve as excellent benchmarks for the sampling of energy landscapes, global optimization, and rare-event kinetics [98100]. One concludes that disorder profoundly reshapes the energy landscape of magnetic nanostructures by transforming good structure-seeking NP ensembles into bad structure-seeking glassy systems.

\section{Disconnectivity graphs}

A very interesting complementary perspective in order to understand the energy landscapes of nanoparticle 


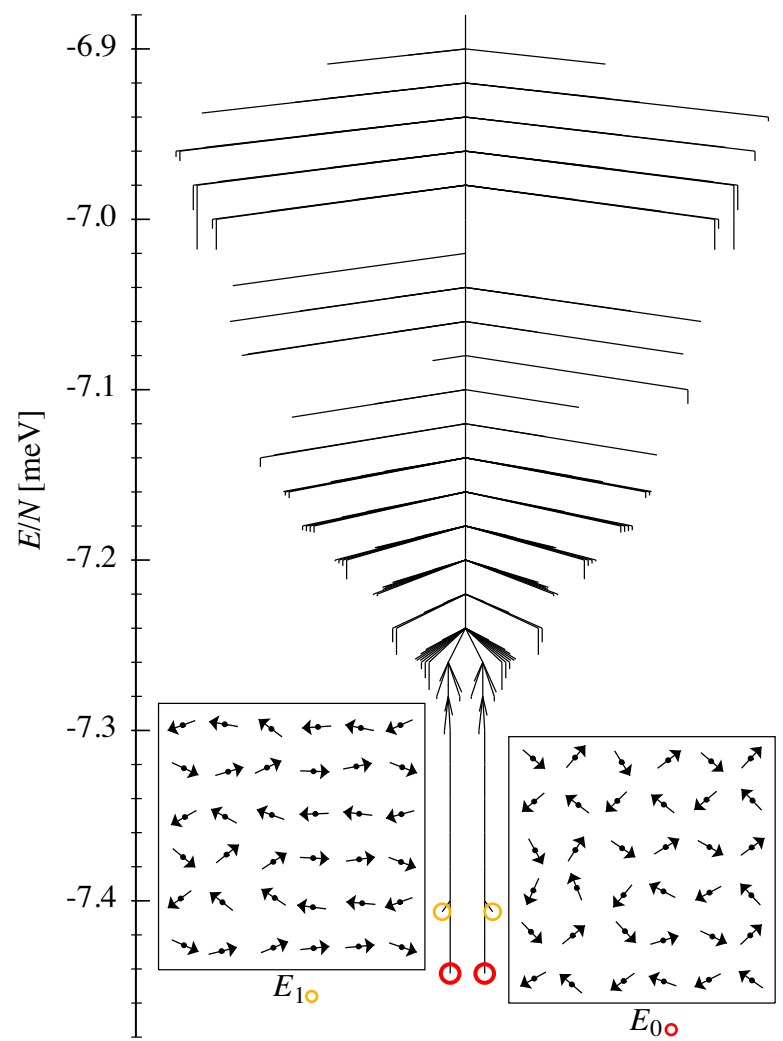

FIG. 5. Disconnectivity graph of the weakly disordered, squarelattice ensemble illustrated in Fig. 1(a). Notice that the degenerate global minima (red circles) and hubs (orange circles) are distinctly more stable than all other local minima. The insets illustrate the magnetic configuration at one of the global minima $\left(E_{0}\right)$ and the closest hub $\left(E_{1}\right)$. The twofold degeneracy is a consequence of time-inversion symmetry. See also Fig. 2 [95].

ensembles is provided by the disconnectivity graphs shown in Figs. 5-7. As discussed in Sec. II C, the focus here no longer resides in the kinetic network of LM but in the energies of the metastable states, taking into account the energy barriers to be surmounted along the connecting MEPs. In Fig. 5, the disconnectivity graph of a weakly disordered square-lattice ensemble is shown. One easily recognizes the degenerate ground states as well as the additional hubs, which lie at a close-by energy and are separated from the former by a very small energy barrier. The strong asymmetry of the energy profiles along the MEPs is also worth noting. Indeed, energy barriers from the excitations to the ground states and hubs are far smaller than those involved in the reversed transitions. Therefore, starting from any given initial state, the system is expected to relax quite rapidly into one of the two ground-state configurations or hubs, even at relatively low temperatures.

The insets of Fig. 5 illustrate the magnetic order in one of the ground states and in the first metastable minimum connected to it, which is also an important hub of the kinetic network (see also Fig. 2). Although the details of the moment orientations depend on the precise location of

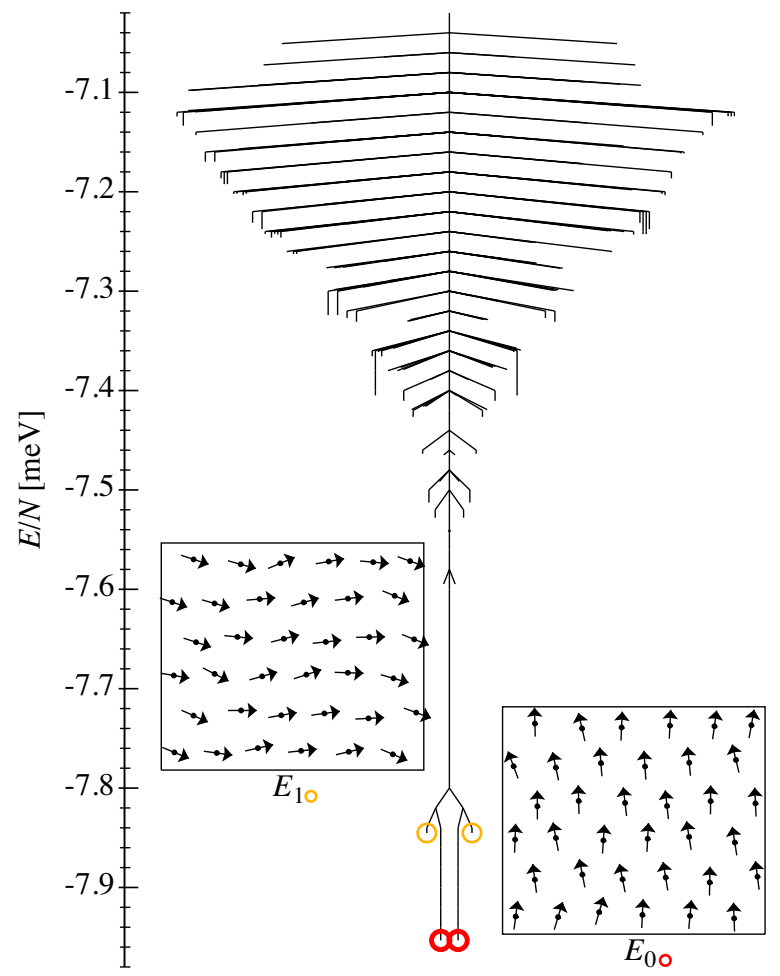

FIG. 6. Disconnectivity graph of the weakly disordered, triangular-lattice ensemble illustrated in Fig. 1(b). As in the square lattice (Fig. 5), the doubly degenerate global minima (red circles) and hubs (orange circles) can be clearly distinguished from the remaining higher-lying local minima, which show particularly small energy barriers towards the ground states. The insets illustrate the magnetic configuration at a global minimum $\left(E_{0}\right)$ and its closest hub $\left(E_{1}\right)$. See also Fig. 3 [95].

the NPs, a number of important features are common to the large majority of weakly disordered square systems. One observes that the ground state is a strongly noncollinear MV state showing flux closure within the NN square plackets. In fact, according to the order parameters introduced in Ref. [32], the ground state shown in Fig. 5 corresponds to $98 \%$ of the perfect periodic MV state. This result is consistent with the work of Prakash et al., who predicted that disorder stabilizes a noncollinear MV configuration [28]. The first metastable states and major hubs are issued from the same continuously degenerate MV ground-state manifold of the periodic case. However, they are qualitatively different from the ground state in the sense that they are closer to the collinear staggered-striped state (see Fig. 5). According to the order parameters introduced in Ref. [32], the hubs in Fig. 5 correspond to $93 \%$ of the perfectly alternating striped state. A further point of considerable interest is to compare the volume in spinconfigurational space of these two remarkable low-lying states since the associated entropy should give us insight on their relative stability at finite $T$. Indeed, one obtains that the local curvatures of the EL at the first metastable hubs are significantly smaller than at the ground state, which 

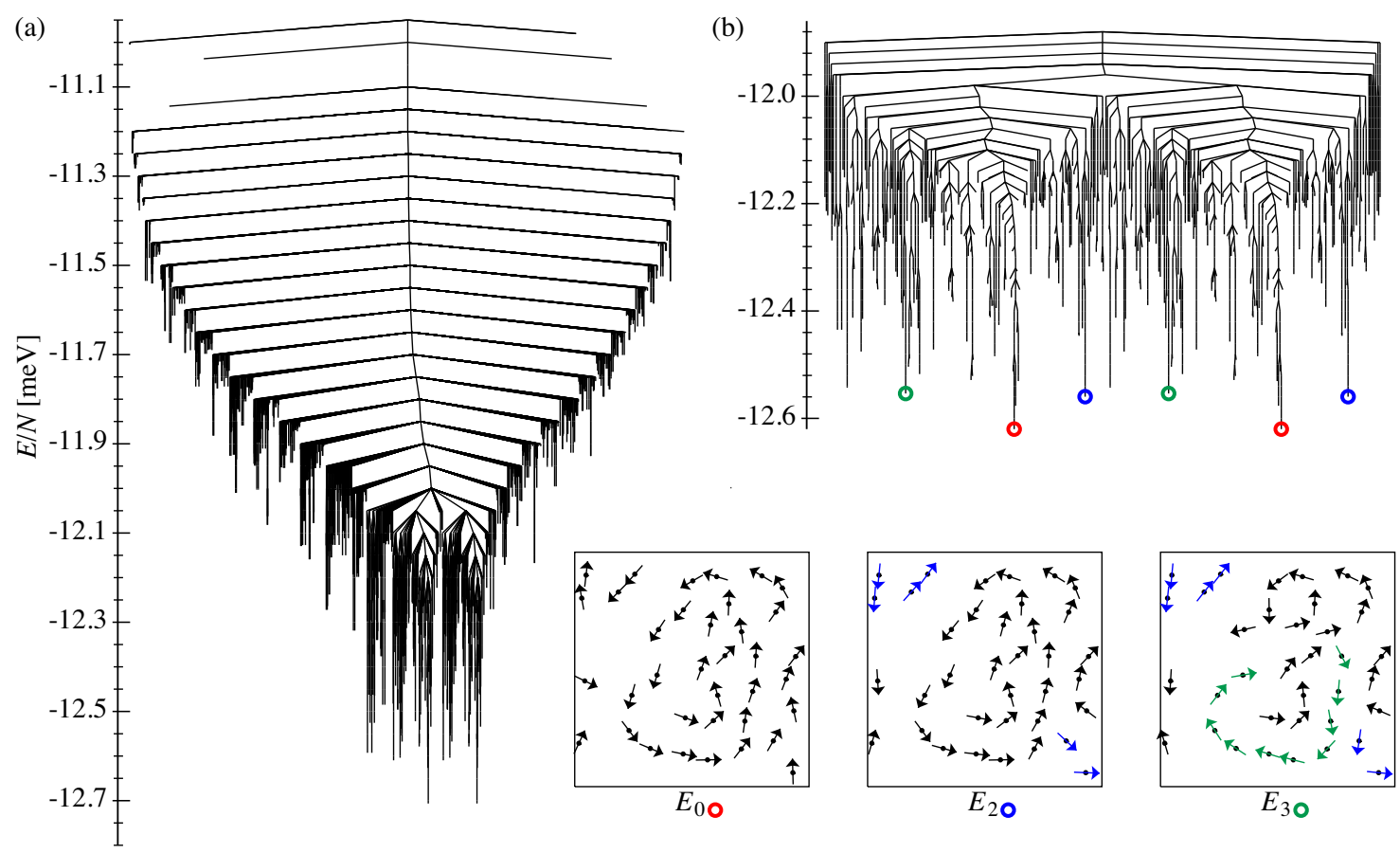

FIG. 7. Disconnectivity graph of the strongly disordered ensemble of nonoverlapping NPs illustrated in Fig. 1(c). In graph (a), all calculated LM are taken into account, whereas graph (b) focuses on the lowest 400 LM. Notice the numerous low-lying local minima, which are separated by important energy barriers that are often much larger than the energy differences between the LM. The energy barriers from the metastable minima towards the low-lying superbasins tend to decrease as the excitation energy increases. The insets illustrate the magnetic configuration in the ground state $\left(E_{0}\right)$ as well as the second $\left(E_{2}\right)$ and third $\left(E_{3}\right)$ excited configurations. The first excited configuration (not shown) is very similar to the ground state [95].

implies a higher local density of states and a larger entropy in the harmonic approximation. This conclusion agrees with the temperature-induced MV-to-AF transition predicted in Ref. [32] as a function of increasing $T$.

The disconnectivity graph corresponding to the weakly disordered triangular ensemble shown in Fig. 6 is qualitatively very similar to the square-lattice one. As in Fig. 5, one finds that the ground states and additional hubs have similar energies and are much more stable than any other excited configuration. Moreover, the energy barriers around the metastable states leading to the ground states or hubs are all very small. These types of DGs (Figs. 5 and 6) correspond to good structure seekers and are usually referred to as palm-tree-like [41,67]. Quantitatively, the ground-state energy per particle $E_{0}$ and the barrier $\Delta E$ separating the ground states are similar to the values obtained for the weakly-disordered square lattice: $E_{0}=$ $-7.84 \mathrm{meV}$ and $\Delta E=0.14 \mathrm{meV}$ in the triangular case, while $E_{0}=-7.44 \mathrm{meV}$ and $\Delta E=0.20 \mathrm{meV}$ in the square case. Furthermore, the dynamically relevant energy barriers at the metastable states have similar heights. The parallelism suggests that in these weakly disordered structures, the symmetry of the underlying periodic structure (square or triangular) and the corresponding ground-state magnetic order (microvortex or ferromagnetic) do not qualitatively affect the topography of the magnetic energy landscape and the resulting relaxation dynamics. These similarities are probably a consequence of the continuous degeneracy of ground-state magnetic order in the perfectly periodic case, which not only can be easily lifted by disorder but also implies that the ground-state basin of attraction is particularly large. Further studies for other periodic-lattice symmetries are necessary before this analysis can be generalized. Among other options, systematic studies of weakly disordered honeycomb and kagome lattices seem particularly worthwhile since the former show a continuous ground-state degeneracy in the periodic case while the latter do not $[28,101-103]$.

Concerning the low-lying magnetic configurations illustrated in the insets of Fig. 6, one observes that both the ground state and the first metastable hub preserve the FM long-range order of the continuously degenerate periodic ground state from which they are issued. The same holds for the transition state connecting them. In general, disorder singles out a few magnetization directions in the low-lying metastable states, with only minor, though clearly appreciable, local deviations in the direction of $\vec{\mu}_{k}$ at different NPs [28,82-84]. As in the weakly disordered square lattice, the long-range magnetic correlations of the periodic case are preserved to a large extent. In the present case, we obtain that the ground state (first metastable hub) matches the order parameter of a perfectly aligned FM state to $98 \%$ (94\%) [see Eq. (12)]. Although the orientations of the NP moments depend on the precise location of the NPs, the 
above-mentioned trends are found to be valid in general, as verified in a large number of different arrangements (about 200), including different numbers of particles and unit cells.

As disorder increases, a completely different physical behavior emerges. Figure 7 shows the disconnectivity graph of the strongly disordered ensemble illustrated in Fig. 1(c). The contrast to the weakly disordered case can hardly be more forceful. If disorder is strong, one finds a large diversity of low-lying metastable states whose energies are close to the ground state. Some of these low-lying configurations are illustrated in the insets. As already observed in previous studies [25-27], they show a strong noncollinearity and a clear tendency toward head-to-tail flux closure (see Fig. 7). Moreover, the changes in spin configuration between different low-lying states involve highly correlated cooperative rearrangements of a limited number of NP moments. The localized character of the spin rearrangements in the strongly disordered ensembles contrasts with the delocalized nature of the elementary LM-TSLM transitions found in the case of weakly disordered square and triangular lattices (cf. the insets of Figs. 5-7). This behavior is in agreement with the previously observed localization of the elementary transitions with an increasing degree of disorder [25-27] and is consistent with the strong increase in the number of local minima and disaggregation of the kinetic networks discussed in Sec. III B.

Although the energy barriers at the high-energy minima remain quite small, those separating the low-lying configurations are quite large, much larger than the energy differences between them. Consequently, the relaxation from high-energy states towards the low-lying states should still be rather fast [96]. However, the thermalization among the low-lying states and the approach to equilibrium should happen on much longer timescales. Qualitatively, the DG in Fig. 7 has many features in common with the banyantree-like graphs [67], particularly at low energies. It closely resembles those found for structural glasses [104-108], which suggests that, in the limit of strong disorder, the ensembles of NPs having weak local anisotropy should behave like superspin glasses. A more detailed comparison with other complex systems is presented in Sec. III D.

Consider a magnetic NP ensemble in some initial highenergy configuration and follow its relaxation towards equilibrium at a given finite temperature. In the case of weakly disordered ensembles, we know from the connectivity analysis that the dynamically relevant transitionsthose with the lowest energy barriers-lead directly or almost directly to one of the ground-state configurations (see Figs. 2 and 3). Moreover, the disconnectivity graphs shown in Figs. 5 and 6 tell us that the energy barriers to overcome in these transitions are much smaller than the energy barriers in the reverse direction. This implies a very high net transition rate from the excited states towards the ground states. Consequently, weakly disordered ensembles should behave like good structure seekers with a fast relaxation dynamics even at pretty low temperatures. In contrast, in the case of strongly disordered NP ensembles, there are no such unhindered relaxation pathways anymore. In fact, the actual relaxation path depends on the initial excited state, which can lead to a number of different magnetic configurations. The disconnectivity graph of Fig. 7 shows quite clearly that the energy barriers around a given metastable state strongly increase as the energy of this state decreases. Particularly, at the bottom of the spectrum, where many low-lying magnetic configurations are present, the energy barriers are much larger than the energy differences between them. This suggests the appearance of multiple timescales along the dynamics, with relatively large transition rates at the early stages of the relaxation (high energies) and a progressive slowing down as equilibrium is approached (low energies) [96], in agreement with the previous analysis of the kinetic networks (Sec. III B). One may therefore conclude, quite generally, that the ensembles of magnetic NPs undergo a transition from good-structure-seeking to glassy behavior as the degree of structural disorder increases.

In order to verify a number of conclusions and assess the dependence of the results on the size of the unit cells, we have performed the energy landscape analysis for systems having unit cells of $N=64$ particles with varying degrees of disorder. The major consequence of considering larger unit cells is a strong increase in the number of LM and TS irrespectively of the degree of disorder. This increase is, in fact, expected to be exponential, at least in the thermodynamic limit where statistical independence can be assumed $[109,110]$. However, the overall topography of the energy landscapes is not qualitatively altered. For example, in the case of weakly disordered ensembles and despite the much larger total number of local minima, there are still only very few local minima around the lowest energy; they define a single clear funnel and act as network hubs. In contrast, for strongly disordered systems, the number of low-lying local minima separated by large energy barriers increases significantly. We therefore conclude that the characteristic properties of the energy landscapes of magnetic NP ensembles - and, in particular, the transition from good to bad structure-seeking behavior as a consequence of increasing disorder-are not qualitatively affected by the size of the unit cell.

Before closing this section, let us observe that the energies of the most stable local minima are significantly lower in strongly disordered ensembles than in weakly disordered systems. This trend is consistent with the previously reported decrease of the average dipole energy of the ensembles with an increasing degree of positional disorder [26]. It can be qualitatively explained in terms of the distance dependence of the dipole interaction and its statistical distribution. When the distance $r_{i j}$ between two particles $i$ and $j$ is randomly varied around a given average value $r_{0}$ defined by the surface coverage, one finds 
that $\left\langle-1 / r_{i j}^{3}\right\rangle \leq-1 / r_{0}^{3}$. Moreover, the difference between $\left\langle-1 / r_{i j}^{3}\right\rangle$ and $-1 / r_{0}^{3}$ increases with increasing interparticledistance variance $\sigma_{r}^{2}$. For the same reason, the landscapes are stretched along the energy axis as the ensembles become increasingly disordered (cf. Figs. 5-7).

\section{Comparison with related energy landscapes}

The results discussed in previous subsections have revealed most remarkable qualitative changes in the properties of the energy landscapes of magnetic nanostructures as a function of the degree of disorder in the NP arrangements. This transformation concerns not only the way in which the metastable magnetic configurations and basins are connected with each other and with the ground state, as reflected by the kinetic networks discussed in Sec. III B, but also the energy distribution of the local minima and transition states, as shown in the disconnectivity graphs of Sec. III C. It is therefore interesting to compare the internal organization of ELs of the nanoparticle ensembles with those of other archetypal complex systems and to identify analogies and specificities.

The ELs of spin systems have previously been investigated in the framework of the classical $X Y$ model with $\mathrm{NN}$ interactions [91]. Despite their common emphasis on the magnetic degrees of freedom, the basic physical characteristics of disordered ensembles of magnetic NP are quite different from those described by the $X Y$ model. The latter concerns spins interacting through $\mathrm{NN}$ ferromagnetic couplings on a periodic lattice and therefore lacks any competing interactions or disorder. The intrinsic continuous 2D spin-rotational symmetry is artificially removed for the calculations by keeping one spin fixed [91]. In contrast, the present NP ensembles involve dipolar magnetic interactions, which are anisotropic, long ranged, and frustrating. Moreover, the spin-rotational degeneracy often found in the periodic NP arrangements is naturally removed by the structural disorder that is inherent to the nanostructure preparation. Nevertheless, it is interesting to observe that the EL of the $X Y$ model corresponds to an efficient relaxation towards the global minimum, i.e., to a good structure seeker. This behavior is qualitatively similar to what we observe in weakly disordered NP ensembles, particularly in the case of triangular ensembles, which show a FM ground state. Furthermore, the downhill energy barriers separating the metastable minima and the lowerlying spin arrangements are, in both cases, very smallsomewhat smaller in the $X Y$ model. The trend to larger barriers is probably a consequence of the long-range and partly frustrating nature of the dipolar couplings among the magnetic NPs, which lead to more complicated cooperative rearrangements of the local moments along the MEPs.

The ferromagnetic nearest-neighbor $X Y$ model is, as expected, incapable of describing the glasslike behavior found in strongly disordered NP ensembles. However, simple, classical Ising models with random interactions, such as the Edward-Anderson and Sherrington-Kirkpatrick models, have attracted a great deal of attention in the context of spin glasses $[40,111,112]$. Although the discrete Ising models are difficult to compare with the present continuous vector model of NP ensembles, some indications of glasslike disconnectivity graphs have been reported [113-115]. Therefore, it would be very interesting to determine the kinetic networks and disconnectivity graphs of $X Y$-like and Heisenberg-like spin-glass models, for different degrees of disorder and parameter regimes, and to compare the results with the present calculations.

Molecular clusters bonded by electric dipolar and quadrupolar interactions are governed by anisotropic, potentially frustrating couplings in a similar way as the magnetic nanoparticle ensembles considered in the previous sections. It is therefore interesting to compare our results with their structural properties and underlying potential energy landscapes, which have been determined in Refs. [92,116]. Several similarities, as well as some qualitative differences, may be observed. Small dipolarbonded clusters are found to be good structure seekers, mainly single-funneled or double-funneled, which is qualitatively similar to the results found in weakly disordered square or triangular magnetic NP ensembles. Moreover, it has been shown that the number of minima in the energy landscape of the clusters increases when the dipolar interactions are switched on, provided that they remain weak so that the optimal structures are not significantly modified with respect to the purely Lennard-Jones case. This has been explained in Ref. [116] as the appearance of multiple arrangements of the electric dipoles within the clusters, which are separated from the low-lying minima by small energy barriers. An analogous behavior is observed in weakly disordered ensembles, as discussed in Sec. III C.

As the strength of the dipole couplings in the clusters is increased, more complex energy landscapes and larger energy barriers between the local minima are found along the relaxation path leading to the global minimum [116]. Still, no evidence is found for the kind of glasslike behavior observed in strongly disordered NP arrangements, revealing some noteworthy differences in the underlying physics. In the case of magnetic NP ensembles, it is experimentally relevant to investigate the energy landscape and the dynamics as a function of the orientation of the magnetic moments, keeping the geometrical arrangement of the NPs fixed. In contrast, in dipolar-bonded clusters, it is important to allow for repositioning of the molecules carrying the dipoles, until the total interatomic forces vanish. Consequently, dipolar clusters tend to modify the local molecular environment by changing their geometry in order to avoid major frustrations of the dipolar couplings. For example, the clusters adopt nearly unfrustrated head-to-tail ring structures when the dipolar interactions are strong enough [116]. As a result, the energy landscapes of the dipolar-bonded clusters are much 
less intricate than those of strongly disordered magnetic NP ensembles or structural glasses.

Previous theoretical studies of glass formers have shown that the energy landscapes of glasses are highly frustrated, showing many LM that are separated by large energy barriers [104-108]. Moreover, no signs of an organization of the landscape in a few dominating funnels can be recognized. As a result, a rapid cooling of the system from a high-temperature disordered state almost inevitably leads to trapping in a more or less limited region of its configurational space corresponding to an amorphous state. Even though a global minimum representing the crystal structure of the solid exists, the supercooled liquid is not able to reach it on the experimental or simulation timescale $[107,108]$. Our results for strongly disordered magnetic NP ensembles (Figs. 4 and 7) display, quite clearly, all of the above-described glassy features. There is neither a clear global minimum nor any identifiable funnel organization. The barrier heights around the low-lying LM are very large, particularly in comparison with the LM-energy differences. However, it is also interesting to note that the strongly disordered NP ensembles do not show any crystal-like, distinctive, global minimum at all. This feature is in contrast with most structural glass formers, for which a crystalline state does exist somewhere in the landscape [104]. If the NP positions were allowed to relax in 2D and the magnetic NP ensemble would adopt a weakly disordered arrangement, the emergence of a crystal-like, dominant, global minimum can be expected (see Figs. 5 and 6).

Another interesting characteristic of structural glasses is the possibility that the particles in the supercooled liquid get trapped within the cage defined by its nearest neighbors. This aspect has been shown to be important since caging restricts the mobility and the diffusion dynamics, particularly at low temperatures. A recent analysis of the energy landscape of a molecular glass former has shown that cagebreaking transitions play a central role in the connectivity of the network of metastable states, thus confirming their importance for diffusion [108]. In the case of magnetic NP ensembles, no clear separation in diffusive and nondiffusive transitions involving different barrier heights could be observed. The distribution of energy barriers is not only broad and rather continuous, but in addition, there is no simple correlation between the change in spin configuration and the height of the barrier separating the metastable states. Overcoming small barriers can lead to both small and very important spin rearrangements. Examples of the latter are the transitions from high-energy to low-energy local minima (see Figs. 5-7). These differences with structural glasses are most probably a consequence of the fact that, in spin systems, there are no inaccessible regions in the configurational space, where the energy essentially diverges (excluded volume). In contrast, in molecular systems, short-distance repulsions result in a relatively clear separation of the energies involved in diffusive and nondiffusive transitions. Although not significant for the dynamics, it is interesting to observe that magnetic NP ensembles also show some transitions where the barrier energy and MEP length are substantial but the configurational change between the local minima is very small or even strictly zero (loop MEP). Finally, one may note that a good correlation is generally found between the cumulative LM-TS-LM distance, which gives a rough measure of the length of the MEP, and the average of the uphill and downhill barriers.

\section{E. Markovian relaxation dynamics}

In order to clarify the relation between the properties of the ELs for the different degrees of disorder and the actual relaxation dynamics of the nanostructures, we have performed numerical simulations of the Markovian dynamics of the ensembles illustrated in Fig. 1. Two complementary physical situations or protocols have been followed in order to prepare the initial probability distribution $p_{i}(0)$ (see Sec. IIE). In the first one, which we refer to as relaxation after quenching (RAQ), the system is first thermalized at a temperature $T^{*}=750 \mathrm{~K}$, which is clearly larger than the energy of all metastable states $\left(k_{B} T^{*}=2.15 \mathrm{meV}\right.$, see Figs. 5-7). At time $t=0$, the system is rapidly quenched to a low temperature $T$, which is in the range of the interesting energy differences and energy barriers of the system and which is kept constant throughout the simulation. In the present case, typical values of $T$ are in the range of $5 \mathrm{~K} \leq T \leq 30 \mathrm{~K}$. This simulation protocol corresponds to a symmetric starting configuration, in which the occupation probabilities of each state are defined by the MaxwellBoltzmann distribution at $T^{*}$. In the second protocol, we simulate an isothermal relaxation after saturation (RAS). Starting from a fully polarized configuration along an arbitrary direction, the external field is removed at time $t=0$, so the system falls into the nearest $\operatorname{LM} j$ of the fieldfree EL. Note that the initial magnetic configuration of the LM $j$ is not fully polarized in general, since it involves a barrierless relaxation starting from the nearest fully polarized state. This case corresponds to an asymmetric configuration, in which the initial occupation probability $p_{i}(0)=\delta_{i j}$ of the $\mathrm{LM} i$ is zero, except for one magnetic state $j$, which is defined by the saturating magnetic field direction.

In order to quantify the time dependence of the longrange magnetic order, we introduce the FM order parameter

$$
\eta_{\mathrm{FM}}=m_{x}^{2}+m_{y}^{2},
$$

where

$$
\vec{m}=\frac{1}{N \mu} \sum_{k} \vec{\mu}_{k}
$$

is the average magnetization of the system measured in units of the NP moment $\mu$. The FM order parameter is 
useful for analyzing the behavior of the triangular and random ensembles. In the case of the square-lattice ensembles, it is more meaningful to follow the time dependence of the MV order parameter

$$
\eta_{\mathrm{MV}}=\tilde{m}_{x}^{2}+\tilde{m}_{y}^{2}
$$

where

$$
\tilde{m}_{x}=\frac{1}{N \mu} \sum_{k l}(-1)^{l} \mu_{k l}^{x}
$$

and

$$
\tilde{m}_{y}=\frac{1}{N \mu} \sum_{k l}(-1)^{k} \mu_{k l}^{y}
$$

are the components of a staggered magnetization adapted to the MV state [29]. In Eqs. (15) and (16), $\mu_{k l}^{x}\left(\mu_{k l}^{y}\right)$ is the $x(y)$ component of the magnetic moment of the NP located at the $k$ th row and $l$ th column of the square arrangement. Notice that $\eta_{\mathrm{MV}}=1$ for any MV state, irrespectively of the MV angle $\alpha$, and that $\eta_{\mathrm{MV}}=0$ in a fully polarized FM state or in a fully disordered infinite sample.

A further interesting property in this context is the configurational entropy of the system given by

$$
S=-k_{B} \sum_{i=1}^{N_{\mathrm{LM}}} p_{i} \ln p_{i}
$$

where $p_{i}$ is the occupation probability of the metastable state $i$. Notice that $S$ represents the entropy of the probability distribution $\left\{p_{i}\right\}$, which, in general, depends on time according to the master equation (8). The entropy associated with the fluctuations of the NP moments $\vec{\mu}_{k}$ within each attraction basin is thereby ignored.

In Fig. 8, the time dependences of the MV order parameter $\eta_{\mathrm{MV}}$ and entropy $S$ of the weakly disordered square ensemble of Fig. 1(a) are displayed for both RAQ and RAS simulations. The dynamics after quenching shows that $\eta_{\mathrm{MV}}$ increases monotonically with $t$, while $S$ decreases. This means that the occupation probabilities of a relatively small number of states with strong MV correlations increase at the expense of most others. One also observes that the equilibrium values of $\eta_{\mathrm{MV}}$ decrease with increasing $T$ since the magnetic configurations with larger $\eta_{\mathrm{MV}}$ are, in general, more stable. At low temperatures $(T \leq 10 \mathrm{~K})$, the relaxation dynamics does not depend strongly on $T$, although a clear trend to faster relaxation is found as $T$ increases $(T \geq 20 \mathrm{~K})$.

In the RAS, the time dependence of $\eta_{\mathrm{MV}}$ is very similar to the RAQ case, even though, from a microscopic perspective, the starting configurations are completely different. However, the entropy $S$ shows a number of

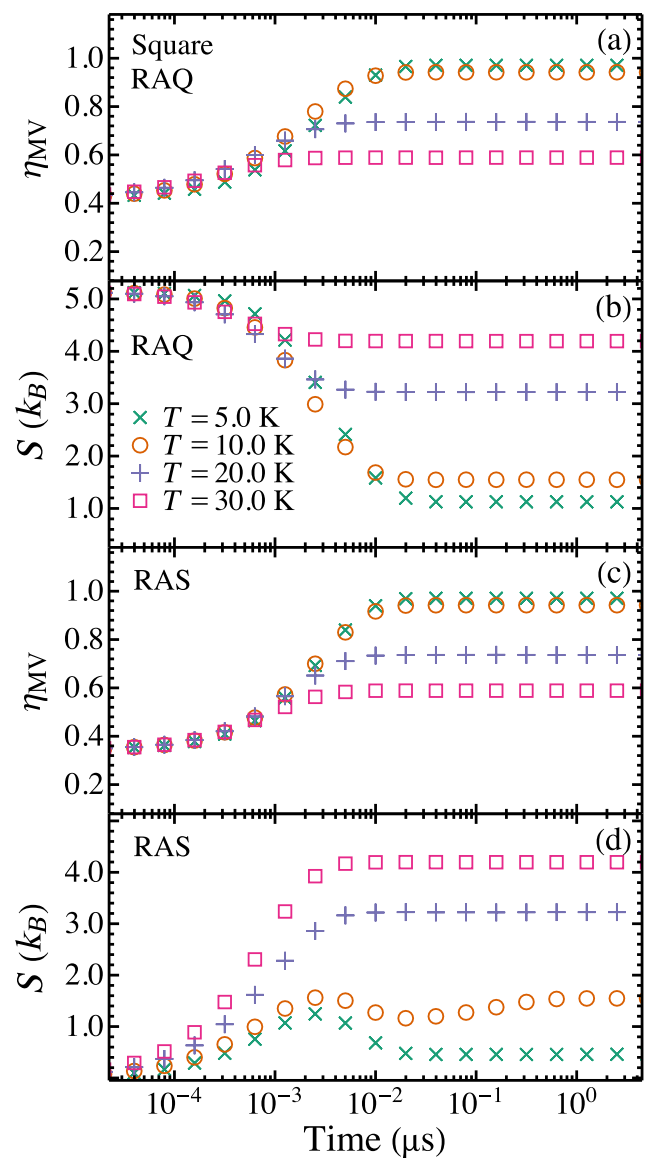

FIG. 8. Time dependence of the MV order parameter $\eta_{\text {MV }}$ and configurational entropy $S$ in the weakly disordered square-lattice ensemble illustrated in Fig. 1(a). The simulations describe the RAQ or the RAS at different temperatures $T$ as indicated.

nonequilibrium features that are not present in the RAQ [see Fig. 8(d)]. Since saturation implies that the system is prepared in a well-defined state, we have $p_{i}(0)=\delta_{i j}$ and $S(0)=0$. When the system relaxes, $S$ increases at first, as the probability distribution $p_{i}$ diffuses out of its initial state and a variety of magnetic configurations are adopted. In this nonequilibrium process and for low temperatures $(T \leq 10 \mathrm{~K}), S$ reaches values that are larger than the equilibrium one [see Fig. 8(d)]. Since the initial saturated state is strongly asymmetric and the dominant transitions from it lead preferentially to one of the ground states (see Figs. 2 and 3), the initial dynamics favors the occupation of one of the ground states rather than the other. This process tends to reduce the configurational entropy at low temperatures. Therefore, $S$ reaches values that are smaller than the equilibrium one at intermediate times [see Fig. 8(d) for $T=10 \mathrm{~K}$ ]. Subsequently, on a longer timescale, the entropy rises again as the system equilibrates between the two degenerate ground states. At very low temperatures, the separation of timescales between the relaxation towards one of the ground states and the overall thermalization is such that $S$ can display a minimum as a function of $t$. 
In Fig. 9, results are presented for the relaxation dynamics of the weakly disordered triangular NP arrangement shown in Fig. 1(b). The time dependences of $\eta_{\mathrm{FM}}$ and $S$ are qualitatively similar to the square-lattice ensemble, with the notable exception that $\eta_{\mathrm{FM}}$ is nearly independent of time in the RAS. The reason for this is that $\eta_{\mathrm{FM}}$ is already very close to its large equilibrium value in the initial saturated state. Moreover, all the dominant states involved in the equilibration process show very strong FM correlations, although the total sample magnetization $\vec{M}$ may point in different directions. As in the RAS simulations for the square-lattice ensembles, the relaxation among the ground states and low-lying configurations with inverted NP moments involves a much longer time than the relaxation towards the ground state that is closest to the initial saturated state. As a result, the time dependence of the entropy $S$ is nonmonotonous at low $T$ [see Fig. 9(d)]. Notice, moreover, that the timescales for the relaxation in the triangular and square ensembles are comparable, although the precise values depend on the energy-barrier heights.

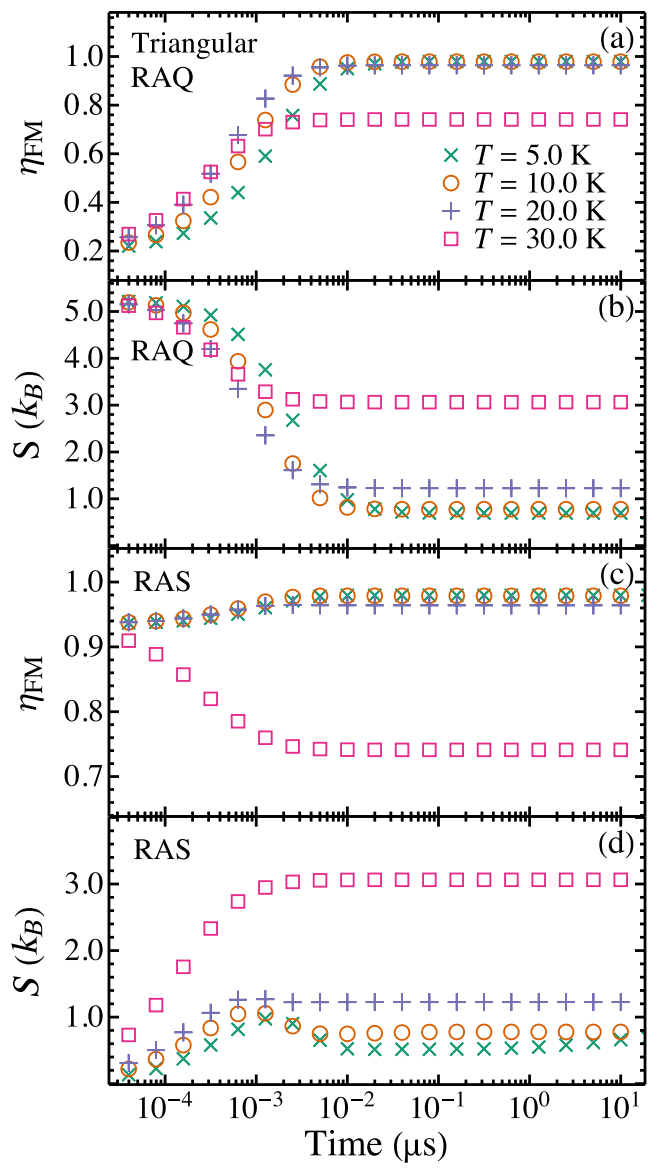

FIG. 9. Time dependence of the FM order parameter $\eta_{\mathrm{FM}}$ and configurational entropy $S$ in the weakly disordered, triangularlattice ensemble illustrated in Fig. 1(b). The simulations correspond to the RAQ or to the RAS at different temperatures $T$ as indicated.
The time dependence of the order parameters $\eta_{\mathrm{MV}}$ and $\eta_{\mathrm{FM}}$ of the weakly disordered ensembles can be approximated by the following stretched exponential law:

$$
\eta(t)=\eta^{\mathrm{eq}}-\left(\eta^{\mathrm{eq}}-\eta^{0}\right) \exp \left[-(t / \tau)^{\beta}\right]
$$

where $\tau$ is the relaxation time, $\beta$ the stretching parameter, $\eta^{0}$ the order parameter at $t=0$, and $\eta^{\text {eq }}$ the order parameter in thermal equilibrium. The fitted values of $\tau$ and $\beta$ for the RAQ and RAS simulations are shown in Table II. One observes, as expected, that $\tau$ decreases as a function of increasing temperature since all transition rates increase. A more detailed analysis shows that at low temperatures ( $T \leq 10 \mathrm{~K}), \tau$ follows approximately an Arrhenius law, i.e., $\ln (\tau) \propto 1 / T$. For higher temperatures, $\ln (\tau)$ decreases faster than proportional to $1 / T$ with increasing $T$, which is sometimes referred to as preArrhenius behavior [117].

The deviations from the purely exponential time dependence, as quantified by the stretching coefficient $\beta$, are interesting since they provide us with a valuable insight on the nature of the relaxation mechanisms. In the large majority of cases, we obtain that $\beta$ is smaller than 1 and that it depends weakly on $T$ (see Table II). Stretched exponential relaxation $(\beta<1)$ means that the dynamics is faster than exponential for short times and slower for long times, which is the consequence of the presence of a distribution of energy barriers [118]. Physically, it can be qualitatively explained as follows: Starting from a highenergy metastable state where the surrounding energy barriers are typically small, the system is, at first, rapidly funneled towards the low-energy spin configurations. Subsequently, once the system reaches the low-energy part

TABLE II. Relaxation time $\tau$ and stretching parameter $\beta$ as obtained by fitting the calculated time dependence of the order parameters $\eta_{\mathrm{MV}}(t)$ of the weakly disordered square ensemble and $\eta_{\mathrm{FM}}(t)$ of the weakly disordered triangular ensemble at different temperatures $T$ [see Eq. (18)]. The first index $s(t)$ refers to the square (triangular) lattice, while the second index $Q(S)$ denotes the relaxation after quenching (saturation). The standard deviations of the fits are very small, in the range $10^{-5}-10^{-3}$.

\begin{tabular}{lcccc}
\hline \hline$T[\mathrm{~K}]$ & 5.0 & 10.0 & 20.0 & 30.0 \\
\hline$\tau_{s, Q}(\mathrm{~ns})$ & 3.31 & 2.09 & 0.83 & 0.34 \\
$\beta_{s, Q}$ & 0.88 & 0.83 & 0.82 & 0.84 \\
$\tau_{s, S}(\mathrm{~ns})$ & 3.14 & 2.83 & 1.59 & 0.97 \\
$\beta_{s, S}$ & 0.96 & 0.93 & 0.94 & 0.93 \\
$\tau_{t, Q}(\mathrm{~ns})$ & 1.99 & 1.07 & 0.67 & 0.37 \\
$\beta_{t, Q}$ & 0.88 & 0.91 & 0.87 & 0.86 \\
$\tau_{t, S}(\mathrm{~ns})$ & 1.52 & 0.81 & 0.45 & 0.38 \\
$\beta_{t, S}$ & 1.00 & 1.00 & 1.00 & 0.80 \\
\hline \hline
\end{tabular}


of the landscape, the energy barriers are larger and the relaxation is slowed down. One concludes that despite the apparent simplicity of the kinetic network of dominant transitions (Figs. 2 and 3) and the palm-tree-like structure [67] of the disconnectivity graphs of weakly disordered ensembles (Figs. 5 and 6), there is still a significant slowing down of the low-temperature dynamics $(\beta<1)$ as compared to the straightforward exponential decay $(\beta=1)$.

For a small number of weakly disordered NP arrangements, more complicated time dependences of the order parameters are observed in the RAS-for example, compressed exponential $(\beta>1)$ or even nonmonotonous behaviors. However, these situations are not representative. In fact, the averages of $\eta_{\mathrm{MV}}$ and $\eta_{\mathrm{FM}}$ over more than 200 different NP arrangements with the same degree of disorder follow Eq. (18) with $\beta \leq 1$. There are two reasons why such anomalies are noticeable in some particular NP arrangements. First, the considered simulation cells that allow an exhaustive characterization of the energy landscape are not large enough to guarantee self-averaging. Second, the initial magnetic state in the RAS is very narrowly defined, which precludes any averaging over different initial basins in the landscape. In this aspect the RAS strongly contrasts with the RAQ of a high-temperature configuration.

Figure 10 shows the relaxation dynamics of a strongly disordered arrangement of NPs [see Fig. 1(c)]. In the RAQ, one observes that $\eta_{\mathrm{FM}}$ is small at all times since the FM order parameter in the initial RAQ state is very close to its nearly vanishing equilibrium value. Furthermore, there is no sign of FM order in the LM involved throughout the relaxation, regardless of their energy. This behavior is consistent with the tendency to flux closure observed in the ground states as well as in the metastable excited configurations [see Fig. 1(c) and the insets of Fig. 7]. Notice that the relaxation of the entropy after quenching, i.e., starting from a fully disordered state, is much slower than in weakly disordered systems [cf. Figs. 8(b), 9(b), and 10(b)]. The approach to equilibrium takes place on a completely different timescale, which is orders of magnitude larger than in the weak disorder situations. It is also important to note that at low temperatures, the relaxation is qualitatively different and much slower than any exponential law. In fact, for $T=5 \mathrm{~K}, S$ decreases almost linearly as a function of $\ln (t)$ over several orders of magnitude, which means that the relaxation timescale grows roughly at the same pace as the simulation time. Such an extremely slowed-down behavior can be regarded as the manifestation of a superspin-glass state.

In the RAS shown in Fig. 10(c), $\eta_{\mathrm{FM}}$ decreases with increasing $t$, which confirms the absence of FM correlations in the low-energy configurations and is consistent with the already-mentioned trend to flux closures. Moreover, notice that the time dependence of $\eta_{\mathrm{FM}}$ is nonmonotonous at all considered temperatures. This feature further demonstrates quantitatively that the equilibration within smaller regions

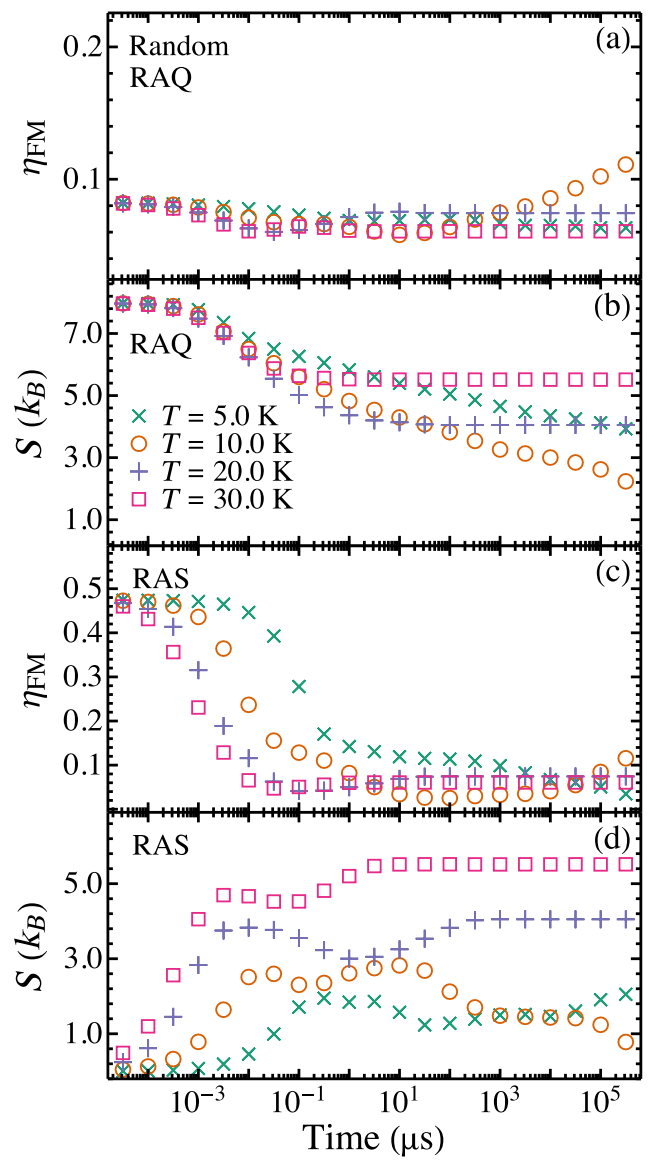

FIG. 10. Time dependence of the FM order parameter $\eta_{\mathrm{FM}}$ and configurational entropy $S$ in the random ensemble of nonoverlapping NPs illustrated in Fig. 1(c). Results are given for the RAQ and for the RAS at different temperatures $T$.

of the configurational space and the wider equilibration between these regions take place at completely different timescales. The remarkably nonmonotonous time dependence of the entropy in the RAS is a clear indication of trapping [see Fig. 10(d)]. Although not completely absent, these subtle dynamical effects tend to soften at the highest considered temperature $(T=30 \mathrm{~K})$ when the energy barriers responsible for trapping can be more effectively overcome within the simulation time.

\section{CONCLUSION}

The ELs of 2D ensembles of dipole-coupled magnetic NPs have been systematically explored. The ergodic connected networks of LM and TS have been determined for various representative NP arrangements. The corresponding kinetic networks and disconnectivity graphs have been derived. A remarkable transformation of the EL has been revealed as a function of the increasing degree of structural disorder. By comparing periodic and weakly disordered square-lattice and triangular-lattice structures, we have shown that even a very weak disorder removes the 
continuous degeneracy of the ground state by singling out a particular magnetic configuration, which preserves the long-range magnetic correlations found in the periodic case (e.g., microvortex-like in the square lattice or ferromagnetic in the triangular lattice). The actual orientations of the NP magnetic moments $\vec{\mu}_{i}$ in the broken-symmetry ground state depend strongly on the particular realization of the nanostructure, bearing no correlation for different disordered arrangements. This consequence of breaking the local point-group symmetry of the lattice can be regarded as an order-by-disorder effect $[83,84,119]$.

A detailed analysis of the topography of the ELs and of the corresponding relaxation dynamics shows that the characteristics of weakly and strongly disordered magnetic nanostructures are profoundly different. For weak disorder, the relaxation processes are funneled towards the groundstate configurations. This mechanism involves very few elementary minimum-saddle-minimum transitions with small energy barriers. The Markovian dynamics is therefore simple, thermally activated, and relatively fast, as in good structure-seeking systems. In contrast, for strong disorder, the relaxation processes comprise a large number of elementary transitions, which are not necessarily directed towards a dominant ground state. The approach to thermal equilibrium requires overcoming increasingly large energy barriers as the energy of the magnetic configuration decreases. The associated rare processes are hindered by trapping, thus leading to multiple characteristic timescales. This behavior is reminiscent of spin glasses and might lead to a breakdown of ergodicity in the thermodynamic limit [39]. Our quantitative simulations of the relaxation dynamics for different degrees of disorder and initial configurations demonstrate that structural disorder profoundly reshapes the collective behavior of magnetic nanostructures by transforming good structure-seeking systems into trapped glassy systems.

An interesting perspective in this context is to systematically follow the evolution of the ELs, as characterized by their kinetic networks, small-world properties, disconnectivity graphs, and magnetic relaxation dynamics as a function of the degree of disorder. On the one hand, these studies would allow us to understand, in more detail, the breaking of translational and point-group symmetry and the lifting of the continuous ground-state degeneracy in the limit of very weak disorder. On the other hand, one is eager to characterize the crossover from good-structure seeker to glassy behavior from a microscopic perspective. Is the transition sharp or smooth as the degree of disorder increases? Should we expect a coexistence between good-structure-seeker behavior at short length scales and glassy behavior at longer length scales for intermediate degrees of disorder? How does this crossover manifest itself in the relaxation dynamics accessible to experiments? Exploring these questions-for example, using the abovediscussed methodology-would boost the experimental and theoretical progress in the field of magnetic nanostructures and motivate further developments.

In the course of this work, a large number of different realizations of the nanostructures have been investigated for different sets of sample parameters. The precise magnetic configurations of the LM and TS, as well as their number, certainly depend on the actual positions of the NPs. However, the general topography of the landscapes is not qualitatively affected by such structural details, provided that the degree of disorder remains the same. Increasing the size of the unit cell (for a fixed coverage) improves the flexibility of the model by allowing a more accurate description of long-range correlations and by reducing the effects of the periodic boundary conditions. Unfortunately, it also implies an exponential increase of the number of LM and TS. For a moderate number of particles $N$ in the cell (e.g., $N=36$ ), it is possible to find essentially all stationary points, which is certainly an advantage with respect to large system sizes, for which one must content oneself with a representative sampling of the landscape. Our results for larger systems (e.g., $N=64$ ) show that increasing the system size does not affect the conclusions on the physical behavior. Despite a remarkable increase of the number of stationary points, the distinctness of the ground state, the connectivity of the kinetic networks, the distribution of energy barriers, etc., remain qualitatively unchanged. These results also suggest that a restricted sampling of a subset of the most relevant LM and TS should accurately reflect the global properties of larger ensembles and should therefore be a valid statistical approach, at least in the present physical context.

The focus of this paper has been on the interparticle interactions and on the cooperative many-body effects resulting from the interplay between interactions and disorder. Still, in view of a comprehensive comparison with experiment, it is important to take into account other, a priori simpler, single-particle contributions to the energy and to quantify how they affect the EL and the dynamics. In this context, one should mention the magnetocrystalline and shape anisotropies of the NPs, which can be tuned experimentally, to some extent, by changing the composition and synthesis procedure. Local uniaxial anisotropies $K_{i}^{(2)}$ introduce an additional, most often aleatory component to the problem, which should tend to favor a more localized, single-particle character of the magnetic excitations. Indeed, larger NP anisotropies imply that the elementary transitions involve fewer particles-in extreme cases, even single-particle magnetization reversals along predefined local easy axes. Thus, interesting changes of the ELs are expected as a function of $K_{i}^{(2)}$ and its statistical distribution.

A further important single-particle contribution of particular experimental and theoretical relevance is the coupling to external magnetic fields [51]. This problem deserves special attention since magnetic fields are usually 
applied to trigger the dynamics. For example, it would be interesting to quantify the magnetization dynamics of the NP ensembles by considering other time-dependent experimental protocols such as frequency-dependent periodic hysteresis loops. External fields are expected to modify the nature of the landscape in a significant way, even if they are static or low frequency, since they tend to remove metastable LM surrounded by small energy barriers and thus lead to catastrophes. Such strong qualitative changes have already been observed in dipolar clusters under the action of external electric fields $[120,121]$. A systematic analysis of the changes in the ELs of disordered NP ensembles as a function of applied magnetic fields is currently in progress.

From a more general perspective, the universality of the above-discussed trends concerning the role of disorder remains an open question, particularly in connection with different types of interparticle couplings and NP arrangements. Let us mention, for instance, the substrate-mediated interactions between NPs deposited on metal surfaces, the exchange interactions between particles having direct metal-metal contacts, and the exchange-bias couplings at interfaces with antiferromagnets. In all of these cases, the essential ingredients for spin-glass behavior, namely, frustration and disorder, happen to be present. Furthermore, as already shown by our own results, the geometrical arrangement of the particles conditions not only the magnetic response but also the way in which it is affected by disorder. Therefore, it is most promising to extend our investigations to other situations of experimental and theoretical interest. In this context, the study of magnetic NP ensembles in disordered kagome and honeycomb lattices deserves special attention since the nature of their ground states is qualitatively different, even in the absence of disorder [28,101-103]. These alternative interactions and lattices could be straightforwardly investigated by applying the calculation and analysis methods presented in this work. Therefore, identifying common features and specificities are a further worth pursuing challenge.

\section{ACKNOWLEDGMENTS}

It is a pleasure to thank Dr. P. J. Jensen for helpful discussions at the early stages of this study and Dr. P. Bessarab for useful comments on his work. One of the authors (D. G.) gratefully acknowledges the Otto-Braun Foundation for support. Computer resources were provided by the IT Service Center of the University of Kassel and by the CSC of the University of Frankfurt.

[1] K. Sattler, J. Mühlbach, and E. Recknagel, Generation of Metal Clusters Containing from 2 to 500 Atoms, Phys. Rev. Lett. 45, 821 (1980).

[2] P. Milani and W. A. deHeer, Improved Pulsed Laser Vaporization Source for Production of Intense Beams of
Neutral and Ionized Clusters, Rev. Sci. Instrum. 61, 1835 (1990).

[3] H. Brune, Microscopic View of Epitaxial Metal Growth: Nucleation and Aggregation, Surf. Sci. Rep. 31, 125 (1998).

[4] S. Sun, Monodisperse FePt Nanoparticles and Ferromagnetic FePt Nanocrystal Superlattices, Science 287, 1989 (2000).

[5] V. F. Puntes, K. M. Krishnan, and A. P. Alivisatos, Colloidal Nanocrystal Shape and Size Control: The Case of Cobalt, Science 291, 2115 (2001).

[6] V. F. Puntes, P. Gorostiza, D. M. Aruguete, N. G. Bastus, and A.P. Alivisatos, Collective Behaviour in TwoDimensional Cobalt Nanoparticle Assemblies Observed by Magnetic Force Microscopy, Nat. Mater. 3, 263 (2004).

[7] L. J. Heyderman, H. H. Solak, C. David, D. Atkinson, R. P. Cowburn, and F. Nolting, Arrays of Nanoscale Magnetic Dots: Fabrication by X-Ray Interference Lithography and Characterization, Appl. Phys. Lett. 85, 4989 (2004).

[8] J. Bansmann, S. H. Baker, C. Binns, J. A. Blackman, J. P. Bucher, J. Dorantes-Dávila, V. Dupuis, L. Favre, D. Kechrakos, A. Kleibert, K. H. Meiwes-Broer, G. M. Pastor, A. Perez, O. Toulemonde, K. N. Trohidou, J. Tuaillon, and Y. Xie, Magnetic and Structural Properties of Isolated and Assembled Clusters, Surf. Sci. Rep. 56, 189 (2005).

[9] C. Binns, K. N. Trohidou, J. Bansmann, S. H. Baker, J. A. Blackman, J.-P. Bucher, D. Kechrakos, A. Kleibert, S. Louch, K.-H. Meiwes-Broer, G. M. Pastor, A. Perez, and Y. Xie, The Behaviour of Nanostructured Magnetic Materials Produced by Depositing Gas-Phase Nanoparticles, J. Phys. D 38, R357 (2005).

[10] C. Desvaux, C. Amiens, P. Fejes, P. Renaud, M. Respaud, P. Lecante, E. Snoeck, and B. Chaudret, MultimillimetreLarge Superlattices of Air-Stable Iron-Cobalt Nanoparticles, Nat. Mater. 4, 750 (2005).

[11] J. V. Barth, G. Costantini, and K. Kern, Engineering Atomic and Molecular Nanostructures at Surfaces, Nature (London) 437, 671 (2005).

[12] P. E. Jönsson, Effects of Interparticle Interaction in Ferromagnetic Nanoparticle Systems, J. Nanosci. Nanotechnol. 10, 6067 (2010).

[13] G.-W. Chern, P. Mellado, and O. Tchernyshyov, TwoStage Ordering of Spins in Dipolar Spin Ice on the Kagome Lattice, Phys. Rev. Lett. 106, 207202 (2011).

[14] A. Farhan, P. M. Derlet, A. Kleibert, A. Balan, R. V. Chopdekar, M. Wyss, L. Anghinolfi, F. Nolting, and L. J. Heyderman, Exploring Hyper-Cubic Energy Landscapes in Thermally Active Finite Artificial Spin-Ice Systems, Nat. Phys. 9, 375 (2013).

[15] S. Zhang, I. Gilbert, C. Nisoli, G.-W. Chern, M. J. Erickson, L. O'Brien, C. Leighton, P. E. Lammert, V.H. Crespi, and P. Schiffer, Crystallites of Magnetic Charges in Artificial Spin Ice, Nature (London) 500, 553 (2013).

[16] S. Anandakumar, T. Andrea, D. Marco, P. Matteo, and V. Paolo, Two-Dimensional Programmable Manipulation of Magnetic Nanoparticles On Chip, Adv. Mater. 26, 2384 (2014).

[17] N. Leo, S. Holenstein, D. Schildknecht, O. Sendetskyi, H. Luetkens, P. M. Derlet, V. Scagnioli, D. Lançon, J. R. L. 
Margegan, T. Prokscha, A. Suter, Z. Salman, S. Lee, and L. J. Heyderman, Collective Magnetism in an Artificial 2D XY Spin System, Nat. Commun. 9, 2850 (2018).

[18] R. Streubel, N. Kent, S. Dhuey, A. Scholl, S. Kevan, and P. Fischer, Spatial and Temporal Correlations of XY Macro Spins, Nano Lett. 18, 7428 (2018).

[19] N. A. Frey and S. Sun, Magnetic Nanoparticle for Information Storage Applications, in Inorganic Nanoparticles: Synthesis, Applications, and Perspectives, edited by C. Altavilla and E. Ciliberto (CRC Press, Boca Raton, FL, 2010), Chap. 2, pp. 33-68.

[20] S. Karmakar, S. Kumar, R. Rinaldi, and G. Maruccio, Nano-Electronics and Spintronics with Nanoparticles, J. Phys. Conf. Ser. 292, 012002 (2011).

[21] H. Brune, M. Giovannini, K. Bromann, and K. Kern, SelfOrganized Growth of Nanostructure Arrays on StrainRelief Patterns, Nature (London) 394, 451 (1998).

[22] Y. Kanai and S. H. Charap, Simulation of Magnetic Aftereffect in Particulate Recording Media, IEEE Trans. Magn. 27, 4972 (1991).

[23] S. Mørup and E. Tronc, Superparamagnetic Relaxation of Weakly Interacting Particles, Phys. Rev. Lett. 72, 3278 (1994).

[24] M. A. Załuska-Kotur, Interacting Small-Particle Systems: Modified Local-Mean-Field Model, Phys. Rev. B 54, 1064 (1996).

[25] P. J. Jensen and G. M. Pastor, Dipole Coupling Induced Magnetic Ordering in an Ensemble of Nanostructured Islands, Phys. Status Solidi A 189, 527 (2002).

[26] P. J. Jensen and G. M. Pastor, Low-Energy Properties of Two-Dimensional Magnetic Nanostructures: Interparticle Interactions and Disorder Effects, New J. Phys. 5, 68 (2003).

[27] G. M. Pastor and P. J. Jensen, Elementary Transitions and Magnetic Correlations in Two-Dimensional Disordered Nanoparticle Ensembles, Phys. Rev. B 78, 134419 (2008).

[28] S. Prakash and C. L. Henley, Ordering Due to Disorder in Dipolar Magnets on Two-Dimensional Lattices, Phys. Rev. B 42, 6574 (1990).

[29] K. De'Bell, A. B. MacIsaac, I. N. Booth, and J. P. Whitehead, Dipolar-Induced Planar Anisotropy in Ultrathin Magnetic Films, Phys. Rev. B 55, 15108 (1997).

[30] P. Politi, M. G. Pini, and R. L. Stamps, Dipolar Ground State of Planar Spins on Triangular Lattices, Phys. Rev. B 73, 020405(R) (2006).

[31] S. K. Baek, P. Minnhagen, and B. J. Kim, KosterlitzThouless Transition of Magnetic Dipoles on the TwoDimensional Plane, Phys. Rev. B 83, 184409 (2011).

[32] D. Schildknecht, L. J. Heyderman, and P. M. Derlet, Phase Diagram of Dipolar-Coupled XY Moments on Disordered Square Lattices, Phys. Rev. B 98, 064420 (2018).

[33] T. Jonsson, J. Mattsson, C. Djurberg, F. A. Khan, P. Nordblad, and P. Svedlindh, Aging in a Magnetic Particle System, Phys. Rev. Lett. 75, 4138 (1995).

[34] M. Sasaki, P. E. Jönsson, H. Takayama, and H. Mamiya, Aging and Memory Effects in Superparamagnets and Superspin Glasses, Phys. Rev. B 71, 104405 (2005).

[35] D. Parker, V. Dupuis, F. Ladieu, J.-P. Bouchaud, E. Dubois, R. Perzynski, and E. Vincent, Spin-Glass Behavior in an
Interacting $\gamma-\mathrm{Fe}_{2} \mathrm{O}_{3}$ Nanoparticle System, Phys. Rev. B 77, 104428 (2008).

[36] S. Bedanta and W. Kleemann, Supermagnetism, J. Phys. D 42, 013001 (2009).

[37] M. Suzuki, S. I. Fullem, I. S. Suzuki, L. Wang, and C.-J. Zhong, Observation of Superspin-Glass Behavior in $\mathrm{Fe}_{3} \mathrm{O}_{4}$ Nanoparticles, Phys. Rev. B 79, 024418 (2009).

[38] G. Margaris, M. Vasilakaki, D. Peddis, K. N. Trohidou, S. Laureti, C. Binns, E. Agostinelli, D. Rinaldi, R. Mathieu, and D Fiorani, Superspin Glass State in a Diluted Nanoparticle System Stabilized by Interparticle Interactions Mediated by an Antiferromagnetic Matrix, Nanotechnology 28, 035701 (2017).

[39] R. G. Palmer, Broken Ergodicity, Adv. Phys. 31, 669 (1982).

[40] K. H. Fischer and J. A. Hertz, Spin Glasses, Cambridge Studies in Magnetism (Book 1) (Cambridge University Press, Cambridge, England, 1993).

[41] D. J. Wales, Energy Landscapes: Applications to Clusters, Biomolecules and Glasses, Cambridge Molecular Science (Cambridge University Press, Cambridge, England, 2004).

[42] K. N. Trohidou and M. Vasilakaki, Monte Carlo Studies of Magnetic Nanoparticles, in Applications of Monte Carlo Method in Science and Engineering, edited by S. Mordechai (InTech, Rijeka, Croatia, 2011), Chap. 20, pp. 513-538.

[43] J. L. Dormann, D. Fiorani, and E. Tronc, Magnetic Relaxation in Fine-Particle Systems, Adv. Chem. Phys. 98, 283 (1997).

[44] D. Kechrakos and K. N. Trohidou, Magnetic Properties of Dipolar Interacting Single-Domain Particles, Phys. Rev. B 58, 12169 (1998).

[45] R. W. Chantrell, N. Walmsley, J. Gore, and M. Maylin, Calculations of the Susceptibility of Interacting Superparamagnetic Particles, Phys. Rev. B 63, 024410 (2000).

[46] D. Kechrakos and K. N. Trohidou, Magnetic Properties of Self-Assembled Interacting Nanoparticles, Appl. Phys. Lett. 81, 4574 (2002).

[47] D. V. Berkov, Numerical Calculation of the Energy Barrier Distribution in Disordered Many-Particle Systems: The Path Integral Method, J. Magn. Magn. Mater. 186, 199 (1998).

[48] R. Dittrich, T. Schrefl, A. Thiaville, J. Miltat, V. Tsiantos, and J. Fidler, Comparison of Langevin Dynamics and Direct Energy Barrier Computation, J. Magn. Magn. Mater. 272-276, 747 (2004).

[49] B. Alkadour, J. I. Mercer, J. P. Whitehead, B. W. Southern, and J. van Lierop, Dipolar Ferromagnetism in ThreeDimensional Superlattices of Nanoparticles, Phys. Rev. B 95, 214407 (2017).

[50] D. Fiorani, J. L. Dormann, R. Cherkaoui, E. Tronc, F. Lucari, F. D’Orazio, L. Spinu, M. Nogues, A. Garcia, and A. M. Testa, Collective Magnetic State in Nanoparticles Systems, J. Magn. Magn. Mater. 196-197, 143 (1999).

[51] P. E. Jönsson, S. Felton, P. Svedlindh, P. Nordblad, and M. F. Hansen, Fragility of the Spin-Glass-Like Collective State to a Magnetic Field in an Interacting Fe-C Nanoparticle System, Phys. Rev. B 64, 212402 (2001). 
[52] S. A. Majetich and M. Sachan, Magnetostatic Interactions in Magnetic Nanoparticle Assemblies: Energy, Time and Length Scales, J. Phys. D 39, R407 (2006).

[53] R. P. Tan, J. S. Lee, J. U. Cho, S. J. Noh, D. K. Kim, and Y. K. Kim, Numerical Simulations of Collective Magnetic Properties and Magnetoresistance in 2D Ferromagnetic Nanoparticle Arrays, J. Phys. D 43, 165002 (2010).

[54] M. Varón, M. Beleggia, T. Kasama, R. J. Harrison, R. E. Dunin-Borkowski, V. F. Puntes, and C. Frandsen, Dipolar Magnetism in Ordered and Disordered Low-Dimensional Nanoparticle Assemblies, Sci. Rep. 3, 1234 (2013).

[55] S. Mondal, S. Choudhury, S. Barman, Y. Otani, and A. Barman, Transition from Strongly Collective to Completely Isolated Ultrafast Magnetization Dynamics in Two-Dimensional Hexagonal Arrays of Nanodots with Varying Inter-Dot Separation, RSC Adv. 6, 110393 (2016).

[56] E. C. Stoner and E. P. Wohlfarth, A Mechanism of Magnetic Hysteresis in Heterogeneous Alloys, Philos. Trans. R. Soc. London Ser. A 240, 599 (1948).

[57] J. P. K. Doye and D. J. Wales, The Effect of the Range of the Potential on the Structure and Stability of Simple Liquids: From Clusters to Bulk, from Sodium to $\mathrm{C}_{60}$, J. Phys. B 29, 4859 (1996).

[58] L. J. Munro and D. J. Wales, Defect Migration in Crystalline Silicon, Phys. Rev. B 59, 3969 (1999).

[59] M. A. Miller, J. P. K. Doye, and D. J. Wales, Structural Relaxation in Morse Clusters: Energy Landscapes, J. Chem. Phys. 110, 328 (1999).

[60] J. Nocedal, Updating Quasi-Newton Matrices with Limited Storage, Math. Comput. 35, 773 (1980).

[61] M.E. J. Newman, Networks (Oxford University, New York, 2010).

[62] A.-L. Barabási, Network Science (Cambridge University Press, Cambridge, England, 2016).

[63] R. D. Luce and A. D. Perry, A Method of Matrix Analysis of Group Structure, Psychometrika 14, 95 (1949).

[64] P. Erdös and A. Rényi, On Random Graphs, Publ. Math. 6, 290 (1957).

[65] D. J. Watts and S.H. Strogatz, Collective Dynamics of 'Small-World' Networks, Nature (London) 393, 440 (1998).

[66] O. M. Becker and M. Karplus, The Topology of Multidimensional Potential Energy Surfaces: Theory and Application to Peptide Structure and Kinetics, J. Chem. Phys. 106, 1495 (1997).

[67] D. J. Wales, M. A. Miller, and T. R. Walsh, Archetypal Energy Landscapes, Nature (London) 394, 758 (1998).

[68] P. Hänggi, P. Talkner, and M. Borkovec, Reaction-Rate Theory: Fifty Years after Kramers, Rev. Mod. Phys. 62, 251 (1990).

[69] W. H. Miller, Importance of Nonseparability in Quantum Mechanical Transition-State Theory, Acc. Chem. Res. 9, 306 (1976).

[70] G. H. Vineyard, Frequency Factors and Isotope Effects in Solid State Rate Processes, J. Phys. Chem. Solids 3, 121 (1957).

[71] P. F. Bessarab, V. M. Uzdin, and H. Jónsson, Harmonic Transition-State Theory of Thermal Spin Transitions, Phys. Rev. B 85, 184409 (2012).
[72] P. F. Bessarab, V. M. Uzdin, and H. Jónsson, Size and Shape Dependence of Thermal Spin Transitions in Nanoislands, Phys. Rev. Lett. 110, 020604 (2013).

[73] T. Gilbert, Classics in Magnetics A Phenomenological Theory of Damping in Ferromagnetic Materials, IEEE Trans. Magn. 40, 3443 (2004).

[74] J. Carrey, B. Mehdaoui, and M. Respaud, Simple Models for Dynamic Hysteresis Loop Calculations of Magnetic Single-Domain Nanoparticles: Application to Magnetic Hyperthermia Optimization, J. Appl. Phys. 109, 083921 (2011).

[75] N. G. van Kampen, Stochastic Processes in Physics and Chemistry (North-Holland, Amsterdam, 1981).

[76] A. F. Voter, Introduction to the Kinetic Monte Carlo Method, in Radiation Effects in Solids, edited by K. E. Sickafus, E. A. Kotomin, and B. P. Uberuaga (Springer, Netherlands, Dordrecht, 2007), p. 1.

[77] D. P. Landau and K. Binder, A Guide to Monte Carlo Simulations in Statistical Physics (Cambridge University Press, Cambridge, England, 2014).

[78] P. I. Belobrov, R. S. Gekht, and V. A. Ignatchenko, Ground State in Systems with Dipole Interaction, Zh. Eksp. Teor. Fiz. 84, 1097 (1983).

[79] The energy $E$ of any MV state can be regarded as a quadratic form $E=\vec{\mu}_{0} P \vec{\mu}_{0}$ of the orientation $\vec{\mu}_{0}=(x, y)$ of one of the NP moments. Any other MV state can be obtained by rotating $\vec{\mu}_{0}$ by an arbitrary angle $\phi$, which physically implies rotating $\vec{\mu}_{k}$ by $\phi$ on one sublattice and by $-\phi$ on the other. Here, $C_{4}$ symmetry implies that $P$ commutes with all rotation matrices of the $C_{4}$ group. Since the two-dimensional representation $(x, y)$ is irreducible under the $C_{4}$ group, this implies that $P=\lambda I$, where $I$ is the identity (Schur's Lemma, see Refs. [80,81]). A similar argument applies to the continuous degeneracy of the FM ground state of the triangular lattice, in this case concerning the $C_{6}$ group.

[80] H. Boerner, Darstellungen von Gruppen mit Berücksichtigung der Bedürfnisse der Modernen Physik (Springer, Berlin, 1955).

[81] D. Schildknecht, M. Schütt, L. J. Heyderman, and P. M. Derlet, Continuous Ground-State Degeneracy of Classical Dipoles on Regular Lattices, Phys. Rev. B. 100, 014426 (2019).

[82] J. Villain, R. Bidaux, J.-P. Carton, and R. Conte, Order as an Effect of Disorder, J. Phys. France 41, 1263 (1980).

[83] C. L. Henley, Ordering Due to Disorder in a Frustrated Vector Antiferromagnet, Phys. Rev. Lett. 62, 2056 (1989).

[84] P. A. McClarty, P. Stasiak, and M. J. P. Gingras, Order-byDisorder in the XY Pyrochlore Antiferromagnet, Phys. Rev. B 89, 024425 (2014).

[85] V. M. Rozenbaum, V. O. Ogenko, and A. A. Chı̌ko, Vibrational and Orientational States of Surface Atomic Groups, Sov. Phys. Usp. 34, 883 (1991).

[86] P. Politi and M. G. Pini, Dipolar Interaction between TwoDimensional Magnetic Particles, Phys. Rev. B 66, 214414 (2002).

[87] J. P. K. Doye, M. A. Miller, and D. J. Wales, Evolution of the Potential Energy Surface with Size for Lennard-Jones Clusters, J. Chem. Phys. 111, 8417 (1999). 
[88] M. A. Miller and D. J. Wales, Energy Landscape of a Model Protein, J. Chem. Phys. 111, 6610 (1999).

[89] C. Zhang and R. S. Berry, Time Autocorrelation Function Analysis of Master Equation and Its Application to Atomic Clusters, J. Chem. Phys. 123, 094103 (2005).

[90] J. M. Carr and D. J. Wales, Refined Kinetic Transition Networks for the GB1 Hairpin Peptide, Phys. Chem. Chem. Phys. 11, 3341 (2009).

[91] D. Mehta, C. Hughes, M. Schröck, and D. J. Wales, Potential Energy Landscapes for the 2D XY Model: Minima, Transition States, and Pathways, J. Chem. Phys. 139, 194503 (2013).

[92] J. Hernández-Rojas, D. Chakrabarti, and D. J. Wales, SelfAssembly of Colloidal Magnetic Particles: Energy Landscapes and Structural Transitions, Phys. Chem. Chem. Phys. 18, 26579 (2016).

[93] D. J. Wales, Decoding Head Capacity Features from the Energy Landscape, Phys. Rev. E 95, 030105(R) (2017).

[94] K. Röder, J. A. Joseph, B. E. Husic, and D. J. Wales, Energy Landscapes for Proteins: From Single Funnels to Multifunctional Systems, Adv. Theory Simul. 2, 1800175 (2019).

[95] The details of the kinetic networks and disconnectivity graphs (e.g., the precise number of connections per node or energy barriers) are different for different positions of the NPs within the unit cell. However, systematic calculations on a large number of disordered nanostructures (more than 200 for each of the considered lattices and degrees of disorder) show that the main features of the graphs, on which the analysis and discussion are based and which condition the relaxation dynamics, are independent of the specific realization.

[96] Qualitative results on the relaxation dynamics are discussed in Sec. III E.

[97] J. W. R. Morgan, D. Mehta, and D. J. Wales, Properties of Kinetic Transition Networks for Atomic Clusters and Glassy Solids, Phys. Chem. Chem. Phys. 19, 25498 (2017).

[98] J. P. K. Doye, M. A. Miller, and D. J. Wales, The DoubleFunnel Energy Landscape of the 38-Atom Lennard-Jones Cluster, J. Chem. Phys. 110, 6896 (1999).

[99] F. Calvo, Free-Energy Landscapes from Adaptively Biased Methods: Application to Quantum Systems, Phys. Rev. E 82, 046703 (2010).

[100] D. J. Wales, Surveying a Complex Potential Energy Landscape: Overcoming Broken Ergodicity Using BasinSampling, Chem. Phys. Lett. 584, 1 (2013).

[101] G. O. Zimmerman, A. K. Ibrahim, and F. Y. Wu, Planar Classical Dipolar System on a Honeycomb Lattice, Phys. Rev. B 37, 2059 (1988).

[102] M. S. Holden, M. L. Plumer, I. Saika-Voivod, and B. W. Southern, Monte Carlo Simulations of a Kagome Lattice with Magnetic Dipolar Interactions, Phys. Rev. B 91, 224425 (2015).

[103] M. Maksymenko, V. R. Chandra, and R. Moessner, Classical Dipoles on the Kagome Lattice, Phys. Rev. B 91, 184407 (2015).
[104] T. F. Middleton and D. J. Wales, Energy Landscapes of Some Model Glass Formers, Phys. Rev. B 64, 024205 (2001).

[105] V. K. de Souza and D. J. Wales, Energy Landscapes for Diffusion: Analysis of Cage-Breaking Processes, J. Chem. Phys. 129, 164507 (2008).

[106] V. K. de Souza and D. J. Wales, Connectivity in the Potential Energy Landscape for Binary Lennard-Jones Systems, J. Chem. Phys. 130, 194508 (2009).

[107] S. P. Niblett, V. K. de Souza, J. D. Stevenson, and D. J. Wales, Dynamics of a Molecular Glass Former: Energy Landscapes for Diffusion in Ortho-Terphenyl, J. Chem. Phys. 145, 024505 (2016).

[108] S. Niblett, M. Biedermann, D. J. Wales, and V. K. de Souza, Pathways for Diffusion in the Potential Energy Landscape of the Network Glass Former $\mathrm{SiO}_{2}$, J. Chem. Phys. 147, 152726 (2017).

[109] F. H. Stillinger and T. A. Weber, Packing Structures and Transitions in Liquids and Solids, Science 225, 983 (1984).

[110] D. J. Wales and J. P. K. Doye, Stationary Points and Dynamics in High-Dimensional Systems, J. Chem. Phys. 119, 12409 (2003).

[111] S. F. Edwards and P. W. Anderson, Theory of Spin Glasses, J. Phys. F 5, 965 (1975).

[112] D. Sherrington and S. Kirkpatrick, Solvable Model of a Spin-Glass, Phys. Rev. Lett. 35, 1792 (1975).

[113] P. Garstecki, T. X. Hoang, and M. Cieplak, Energy Landscapes, Supergraphs, and "Folding Funnels" in Spin Systems, Phys. Rev. E 60, 3219 (1999).

[114] H. Seyed-allaei, H. Seyed-allaei, and M. R. Ejtehadi, Energy-Landscape Networks of Spin Glasses, Phys. Rev. E 77, 031105 (2008).

[115] Q. Zhou and W. H. Wong, Energy Landscape of a SpinGlass Model: Exploration and Characterization, Phys. Rev. E 79, 051117 (2009).

[116] J. D. Farrell, C. Lines, J. J. Shepherd, D. Chakrabarti, M. A. Miller, and D. J. Wales, Energy Landscapes, Structural Topologies and Rearrangement Mechanisms in Clusters of Dipolar Particles, Soft Matter 9, 5407 (2013).

[117] H. G. E. Hentschel, S. Karmakar, I. Procaccia, and J. Zylberg, Relaxation Mechanisms in Glassy Dynamics: The Arrhenius and Fragile Regimes, Phys. Rev. E 85, 061501 (2012).

[118] R. G. Palmer, D. L. Stein, E. Abrahams, and P. W. Anderson, Models of Hierarchically Constrained Dynamics for Glassy Relaxation, Phys. Rev. Lett. 53, 958 (1984).

[119] J. Villain, Insulating Spin Glasses, Z. Phys. B 33, 31 (1979).

[120] J. Hernández-Rojas, B. S. González, T. James, and D. J. Wales, Thermodynamics of Water Octamer in a Uniform Electric Field, J. Chem. Phys. 125 (2006).

[121] T. James, D. J. Wales, and J. Hernández-Rojas, Energy Landscapes for Water Clusters in a Uniform Electric Field, J. Chem. Phys. 126 (2007). 